Pacific

Journal of

Mathematics

\title{
SIMILAR FILLINGS AND ISOLATION OF CUSPS OF HYPERBOLIC 3-MANIFOLDS
}

ROBERTO FRIGERIO 


\title{
SIMILAR FILLINGS AND ISOLATION OF CUSPS OF HYPERBOLIC 3-MANIFOLDS
}

\author{
ROBERTO FRIGERIO
}

\begin{abstract}
We deepen the analysis of certain classes $M_{g, k}$ of hyperbolic 3-manifolds that were introduced in a previous work by B. Martelli, C. Petronio and the author. Each element of $\mu_{g, k}$ is an oriented complete finite-volume hyperbolic 3-manifold with compact connected geodesic boundary of genus $\boldsymbol{g}$ and $k$ cusps. We prove that several elements in $M_{g, k}$ admit nonhomeomorphic hyperbolic Dehn fillings sharing the same volume, homology, cusp volume, cusp shape, Heegaard genus, complex length of the shortest geodesic, length of the shortest return path, and Turaev-Viro invariants.

Let $N$ be a complete finite-volume hyperbolic 3-manifold with (possibly empty) geodesic boundary and cusps $C_{1}, \ldots, C_{h}, C_{h+1}, \ldots, C_{k}$. According to Neumann and Reid, the cusps $C_{1}, \ldots, C_{h}$ are said to be geometrically isolated from $C_{h+1}, \ldots, C_{k}$ if any small deformation of the hyperbolic structure on $N$ induced by Dehn filling $C_{h+1}, \ldots, C_{k}$ does not affect the Euclidean structure at $C_{1}, \ldots, C_{h}$. We show here that the cusps of any manifold in $M_{g, k}$ are geometrically isolated from each other. On the contrary, any element in $\mu_{g, k}$ admits an infinite family of hyperbolic Dehn fillings inducing nontrivial deformations of the hyperbolic structure on the geodesic boundary.
\end{abstract}

Let $N$ be an oriented complete finite-volume hyperbolic 3-manifold with compact geodesic boundary. Mostow-Prasad's rigidity Theorem implies that the space (of homotopy classes) of complete finite-volume structures supported by $N$ reduces to a single point, so nontrivial deformations of the complete structure can give rise only to incomplete metrics. It is a well-known fact that such deformations are closely related to the geometry of manifolds which can be obtained from $N$ via Dehn filling, as we are now going to explain.

A slope on a torus is an isotopy class of simple unoriented closed curves. Let $X$ be an oriented 3-manifold with boundary tori $T_{1}, \ldots, T_{k}$ and let $V_{1}, \ldots, V_{h}$ be solid tori, $h \leqslant k$. Let $s_{i}$ be a slope on $T_{i}$ for $i=1, \ldots, h$ and choose an attaching

MSC2000: primary 57M50; secondary 58H15.

Keywords: Dehn filling, geodesic boundary, truncated tetrahedron, Kojima decomposition, commensurability.

Supported by the INTAS project CalcoMet-GT 03-51-3663. 
homeomorphism $\varphi_{i}: \partial V_{i} \rightarrow T_{i}$ taking a meridian of $V_{i}$ onto a loop representing $s_{i}$. Set $\Phi=\left(\varphi_{1}, \ldots, \varphi_{h}\right)$ and $X\left(s_{1}, \ldots, s_{h}\right)=X \cup_{\Phi}\left(V_{1} \cup \cdots \cup V_{h}\right)$. We say that $X\left(s_{1}, \ldots, s_{h}\right)$ is obtained by Dehn filling $X$ along the $s_{i}$. It is easily seen that $X\left(s_{1}, \ldots, s_{h}\right)$ is a 3-manifold whose homeomorphism type depends solely on the $s_{i}$.

A complete finite-volume hyperbolic $N$ admits a natural compactification obtained by adding some boundary tori (with an abuse, we shall often denote also such compactification by the same symbol $N$ ). Thus, it does make sense to consider the Dehn fillings of $N$. A crucial fact is that the metric completions of many small deformations of the complete metric of $N$ actually define complete hyperbolic structures on manifolds obtained by Dehn filling $N$. This phenomenon is at the heart of the proof of Thurston's hyperbolic Dehn filling Theorem [1979], which states that "almost all" the Dehn fillings of a cusped 3-manifold support a complete finite-volume hyperbolic metric.

\section{Preliminaries and statements}

All the manifolds considered in this paper will be connected and oriented. Let $\Delta$ denote the standard tetrahedron, and let $\dot{\Delta}$ be $\Delta$ with its vertices removed. An ideal triangulation of a compact 3-manifold with boundary $M$ is a realization of the interior of $M$ as a gluing of a finite number of copies of $\dot{\Delta}$, induced by a simplicial face-pairing of the corresponding $\Delta$ 's. Let $\Sigma_{g}$ be the closed orientable surface of genus $g$. It is proved in [Frigerio et al. 2003] that an ideal triangulation of a manifold whose boundary is the union of $\Sigma_{g}$ and $k$ tori contains at least $g+k$ tetrahedra. This motivates the following definition of $\mu_{g, k}$, for all $g>k \geqslant 1$ :

$M_{g, k}=\{$ compact oriented manifolds $M$ having an ideal triangulation

$$
\text { with } \left.g+k \text { tetrahedra, and } \partial M=\Sigma_{g} \sqcup\left(\bigsqcup_{i=1}^{k} T_{i}\right) \text { with } T_{i} \cong \Sigma_{1}\right\} \text {. }
$$

We also proved there that any element in $\mu_{g, k}$ admits a complete finite-volume hyperbolic structure with geodesic boundary.

We recall that the valence of an edge in a triangulation is the number of tetrahedra incident to it (with multiplicity). The following result shows that manifolds in $M_{g, k}$ admit combinatorial decompositions with very particular features. As a consequence, we will see in Theorem 2.3 that the geometry supported by such manifolds is very "symmetric".

Proposition 1.1 [Frigerio et al. 2003]. Let $M \in \mathcal{M}_{g, k}$ and suppose that $\mathscr{T}$ is an ideal triangulation of $M$ with $g+k$ tetrahedra.

- For any $i=1, \ldots, k$ there are exactly two tetrahedra of $\mathcal{T}$ with 3 vertices on $\Sigma_{g}$ and one on $T_{i}$; the remaining $g-k$ tetrahedra have all 4 vertices on $\Sigma_{g}$; 
- $T$ has $k+1$ edges $e_{0}, \ldots, e_{k}$ such that $e_{0}$ has both its endpoints on $\Sigma_{g}$ and valence $6 g$, while $e_{i}$ connects $\Sigma_{g}$ to $T_{i}$ and has valence 6 for $i=1, \ldots, k$.

However, it turns out that elements in $\mu_{g, k}$, even if far from being generic, are in a big number. It is proved in [Frigerio et al. 2003] that $\mathcal{M}_{g, k}$ is nonempty for all $g>k \geqslant 1$, and that for any fixed $k$ there exist constants $C>c>0$ such that $n^{c \cdot n}<\# M_{g, k}<n^{C \cdot n}$ for $n \gg 0$.

Isolation of cusps. Recall that if $N$ is a complete finite-volume hyperbolic 3manifold, then every boundary torus of $N$ is naturally endowed with a Euclidean structure, defined up to similarity. Neumann and Reid [1993] introduced the notion of geometric isolation for cusps in a hyperbolic manifold:

Definition 1.2. Let $N$ be a complete finite-volume hyperbolic 3-manifold with (possibly empty) geodesic boundary and cusps $C_{1}, \ldots, C_{h}, C_{h+1}, \ldots, C_{k}$. We say that $C_{1}, \ldots, C_{h}$ are geometrically isolated from $C_{h+1}, \ldots, C_{k}$ if any small deformation of the hyperbolic structure on $N$ induced by Dehn filling $C_{h+1}, \ldots, C_{k}$ while keeping $C_{1}, \ldots, C_{h}$ complete does not affect the Euclidean structure at $C_{1}, \ldots, C_{h}$.

Calegari [2001] described different strategies for constructing manifolds with isolated cusps, also providing explanations for all the previously known examples of isolation phenomena. In Section 3 we show that the cusps of any manifold $M \in \mathcal{M}_{g, k}$ are geometrically isolated from each other:

Theorem 1.3. Let $M \in \mathcal{M}_{g, k}$ with cusps $C_{1}, \ldots, C_{k}$ and let $h \leqslant k$. Then $C_{1}, \ldots, C_{h}$ are geometrically isolated from $C_{h+1}, \ldots, C_{k}$.

Apparently, isolation of cusps in our examples arises for different reasons from those described in [Calegari 2001].

Nonisolation of the boundary. On page 351 we sketch a proof of this result:

Theorem 1.4. Let $M \in \mathcal{M}_{g, k}$. Then there exists an infinite set $\left\{N_{i}\right\}_{i \in \mathbb{N}}$ of complete finite-volume hyperbolic 3-manifolds with the property that each $N_{i}$ is obtained by Dehn filling $M$ and the hyperbolic surfaces $\partial M, \partial N_{1}, \ldots, \partial N_{i}, \ldots$ are pairwise nonisometric.

Examples of isolation of the geodesic boundary from cusps of hyperbolic 3manifolds were provided in [Neumann and Reid 1993; Fujii 1993]. Nonisolation phenomena were described in [Fujii 1992; Fujii and Kojima 1997; Kent 2005].

Similar fillings. Let $N$ be an oriented complete hyperbolic 3-manifold and take a closed geodesic $\ell \subset N$. There exists a well-defined complex length $L_{\mathbb{C}}(\ell) \in$ $\mathbb{C} / 2 \pi i \mathbb{Z}$, which can be described as follows. The real part of $L_{\mathbb{C}}(\ell)$ is simply the 
usual length of $\ell$. Let $x_{0}$ be a point on $\ell$, let $v \in T_{x_{0}}(N)$ be a tangent vector at $x_{0}$ which is perpendicular to $\ell$, and fix an auxiliary orientation on $\ell$. Let $v^{\prime}$ be the vector obtained by parallel transporting $v$ from $x_{0}$ back to $x_{0}$ along $\ell$. Together with the orientation of $\ell$, the orientation of $N$ at $x_{0}$ determines a well-defined signed angle between $v$ and $v^{\prime}$. Such an angle is independent from the choice of $x_{0}, v$, and the auxiliary orientation of $\ell$, and gives the imaginary part of $L_{\mathbb{C}}(\ell)$.

If $N$ is complete finite-volume with compact geodesic boundary and $k$ cusps, the cusp shape of $N$ is the set of Euclidean structures (up to scale factors) induced on the boundary tori of $N$. A regular horocusp neighbourhood for $N$ is a set $O_{1} \sqcup \cdots \sqcup O_{k} \subset N$, where $O_{i}$ is an open embedded horospherical neighbourhood of the $i$-th cusp of $N, O_{i} \cap O_{j}=\varnothing$ for $i \neq j$ and $\operatorname{vol}\left(O_{i}\right)=\operatorname{vol}\left(O_{j}\right)$ for $i, j \in\{1, \ldots, k\}$. The cusp volume of $N$ is the volume of a maximal regular horocusp neighbourhood for $N$ (where this volume is intended to be 0 if $N$ is compact). A return path in $N$ is a geodesic segment in $N$ intersecting $\partial N$ perpendicularly in its endpoints. Since the boundary of $N$ is compact, it is easily seen that there exists a (not necessarily unique) shortest return path in $N$.

If $N$ is a compact 3-manifold with $\partial N=\partial_{0} N \sqcup \partial_{1} N$, one can define the Heegaard genus of $\left(N, \partial_{0} N, \partial_{1} N\right)$ as the minimal genus of a surface that splits $N$ as $C_{0} \sqcup$ $C_{1}$, where $C_{i}$ is obtained by attaching 1-handles on one side of a collar of $\partial_{i} N$. Moreover, for any integer $r \geqslant 2$, after fixing in $\mathbb{C}$ a primitive $(2 r)$-th root of unity, a real-valued invariant $\operatorname{TV}_{r}(N)$ was defined in [Turaev and Viro 1992].

Definition 1.5. Let $N, N^{\prime}$ be complete finite-volume hyperbolic 3-manifolds with geodesic boundary and the same number of cusps. We say that $N$ and $N^{\prime}$ are geometrically similar if the following conditions hold:

- $N$ and $N^{\prime}$ share the same volume, the same cusp volume and the same cusp shape.

- The shortest return paths of $N$ and $N^{\prime}$ have the same length.

- The shortest closed geodesics of $N$ and $N^{\prime}$ have the same complex length.

- $H_{1}(N ; \mathbb{Z}) \cong H_{1}\left(N^{\prime} ; \mathbb{Z}\right)$.

- If $\Sigma$ (resp. $\Sigma^{\prime}$ ) is the geodesic boundary of $N$ (resp. of $N^{\prime}$ ) and $T_{1}, \ldots, T_{k}$ (resp. $\left.T_{1}^{\prime}, \ldots, T_{k}^{\prime}\right)$ are the boundary tori of $N$ (resp. of $N^{\prime}$ ), then the Heegaard genus of $\left(N, \Sigma, T_{1} \sqcup \cdots \sqcup T_{k}\right)$ is equal to that of $\left(N^{\prime}, \Sigma^{\prime}, T_{1}^{\prime} \sqcup \cdots \sqcup T_{k}^{\prime}\right)$.

- $N$ and $N^{\prime}$ have the same Turaev-Viro invariants.

Geometrically similar hyperbolic 3-manifolds were first studied in [Hodgson et al. 1992], where it was shown that the Whitehead link complement admits an infinite sequence of pairs of nonhomeomorphic geometrically similar Dehn fillings (the definition of geometric similarity introduced in that paper is actually a bit different from ours, and regards cusped manifolds without geodesic boundary). 
We show here that if $M \in M_{g, k}$ is generic (meaning that it does not admit too many isometries), we can construct different geometrically similar manifolds by filling $M$ along slopes on any chosen set of cusps of $M$. This allows us to prove the following:

Theorem 1.6. For any $k>0$ there exist $g>k$ and an element $X_{k} \in \mathcal{M}_{g, k}$ with boundary tori $T_{1}, \ldots, T_{k}$ having the following property. For each $i=1, \ldots, k$ there exists a finite set $\mathscr{Y}_{i}$ of slopes on $T_{i}$ such that if $h \leqslant k$ and $s_{i} \notin \mathscr{Y}_{i}$ is a slope on $T_{i}$, then $X_{k}\left(s_{1}, \ldots, s_{h}\right)$ is hyperbolic and at least

$$
\frac{k ! 3^{h}}{h !(k-h) !}
$$

pairwise nonhomeomorphic hyperbolic Dehn fillings of $X_{k}$ are geometrically similar to $X_{k}\left(s_{1} \ldots, s_{h}\right)$.

The elements of any pair of geometrically similar manifolds described in [Hodgson et al. 1992] are commensurable with each other. On the contrary, the geometrically similar manifolds we obtain are typically noncommensurable with each other.

\section{Triangulations and deformation space}

In order to construct a hyperbolic structure on our manifold $M \in \mathcal{M}_{g, k}$ we realize the tetrahedra of an ideal triangulation of $M$ as special geometric blocks in $\mathbb{H}^{3}$ and then we require that the structures match under the gluings.

Geometric tetrahedra. A partially truncated tetrahedron is a pair $(\Delta, \mathscr{I})$, where $\Delta$ is a tetrahedron and either $\mathscr{I}=\varnothing$ or $\mathscr{I}=\{v\}$, where $v$ is a vertex of $\Delta$. In the latter case we say that $v$ is the ideal vertex of $\Delta$. In the sequel we will always refer to $\Delta$ itself as a partially truncated tetrahedron, tacitly implying that $\mathscr{I}$ is also fixed. The topological realization $\Delta^{*}$ of $\Delta$ is obtained by removing from $\Delta$ the ideal vertex, if $\Phi \neq \varnothing$, and small open stars of the nonideal vertices. We call lateral hexagon and truncation triangle the intersection of $\Delta^{*}$ respectively with a face of $\Delta$ and with the link in $\Delta$ of a nonideal vertex. The edges of the truncation triangles, which also belong to the lateral hexagons, are called boundary edges, and the other edges of $\Delta^{*}$ are called internal edges. If $\Delta$ has an ideal vertex, three lateral hexagons of $\Delta^{*}$ are in fact pentagons with a vertex removed, and they are called exceptional lateral hexagons.

A geometric realization of $\Delta$ is an embedding of $\Delta^{*}$ in $\mathbb{M}^{3}$ such that the truncation triangles are geodesic triangles, the lateral hexagons are geodesic polygons with ideal vertices corresponding to missing edges, and truncation triangles and lateral hexagons lie at right angles to each other. The next theorem classifies isometry classes of geometric partially truncated tetrahedra. 
Theorem 2.1 [Fujii 1990; Frigerio and Petronio 2004]. Let $\Delta$ be a partially truncated tetrahedron and let $\Delta^{(1)}$ be the set of edges of $\Delta$. The geometric realizations of $\Delta$ are parameterized up to isometry by the dihedral angle assignments $\theta: \Delta^{(1)} \rightarrow(0, \pi)$ such that for each vertex $v$ of $\Delta$, if $e_{1}, e_{2}, e_{3}$ are the edges that emanate from $v$, then $\theta\left(e_{1}\right)+\theta\left(e_{2}\right)+\theta\left(e_{3}\right)$ is equal to $\pi$ for ideal $v$ and less than $\pi$ for nonideal $v$.

Hyperbolicity equations. Let $M$ be an element of $\mu_{g, k}$ and $\mathscr{T}$ be an ideal triangulation of $M$ with $g+k$ tetrahedra. We try to give $M$ a hyperbolic structure with geodesic boundary by looking for a geometric realization $\theta$ of $\mathscr{T}$ such that the structures of the tetrahedra match under the gluings. In order to define a global hyperbolic structure on $M$, the tetrahedra of $\mathscr{T}$ must satisfy two obvious necessary conditions, which in fact are also sufficient [Frigerio 2006]. Namely, we should be able to glue the lateral hexagons by isometries, and we should have a total dihedral angle of $2 \pi$ around each edge of the manifold.

By Proposition 1.1, if we suppose $M$ to be hyperbolic and $\mathscr{T}$ to be geometric (i. e. to define a hyperbolic structure on the whole of $M$ ), than the edges of the tetrahedra with all the vertices on $\Sigma_{g}$ should have all the same length. This would force the realizations of the compact tetrahedra in $\mathscr{T}$ to be regular and isometric to each other.

On each tetrahedron of $\mathscr{T}$ we fix the orientation compatible with the global orientation of $M$. As a result also the lateral hexagons have a fixed orientation, which is reversed by the gluing maps. We now fix some notation to be used extensively later on. Let $T_{1}, \ldots, T_{k}$ be the boundary tori of $M$. We denote by $\Delta_{2 i-1}, \Delta_{2 i}$ the tetrahedra of $\mathscr{T}$ incident to $T_{i}$ and by

$$
F_{2 i-1}^{1}, F_{2 i-1}^{2}, F_{2 i-1}^{3}, F_{2 i}^{1}, F_{2 i}^{2}, F_{2 i}^{3}
$$

the exceptional hexagons of $\Delta_{2 i-1}, \Delta_{2 i}$, in such a way that $F_{2 i-1}^{j}$ is glued to $F_{2 i}^{j}$ for $j=1,2,3$. For $l=1, \ldots, 2 k$ we also suppose that $F_{l}^{1}, F_{l}^{2}, F_{l}^{3}$ are positively arranged around the ideal vertex of $\Delta_{l}$, and we call $e_{l}^{j}$ the only finite internal edge of $F_{l}^{j}$, and $f_{l}^{j}$ the edge of $\Delta_{l}$ opposite to $e_{l}^{j}$. We now consider a geometric realization $\theta$ of the tetrahedra of $\mathcal{T}$ such that compact tetrahedra are regular and isometric to each other, and for $l=1, \ldots, 2 k, j=1,2,3$ we set $\alpha_{l}^{j}=\theta\left(e_{l}^{j}\right)$, and $\gamma_{l}^{j}=\theta\left(f_{l}^{j}\right)$ (see Figure 1). We set $\beta$ to be the dihedral angle along the edges of the $g-k$ compact tetrahedra of $\mathscr{T}$.

Consistency along the faces. We first determine the conditions on dihedral angles under which all the compact lateral hexagons of the tetrahedra in $\mathcal{T}$ are regular and isometric to each other. This is equivalent to asking that the lengths of all the boundary edges of all the compact lateral hexagons are equal to each other, and 

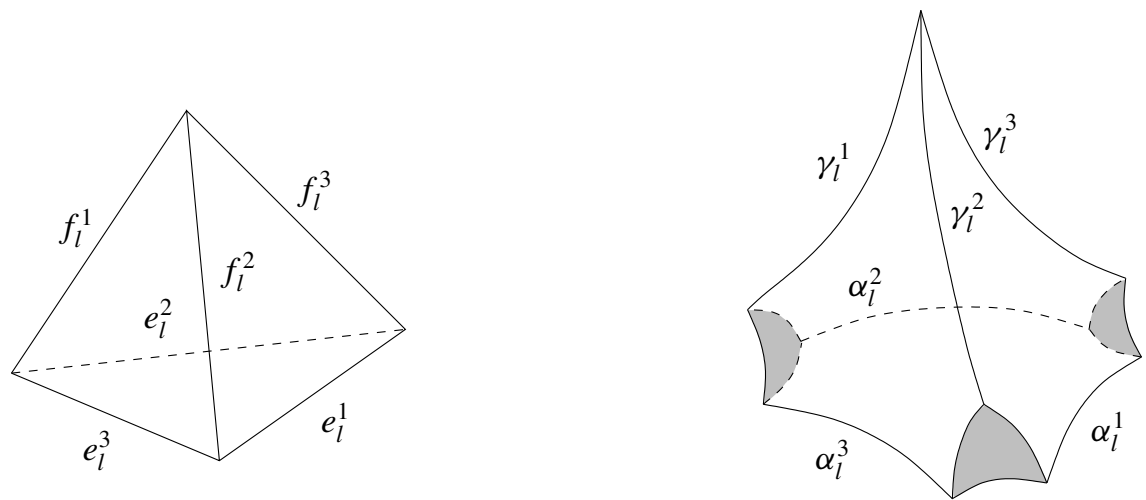

Figure 1. Dihedral angles along the edges of a noncompact tetrahedron of $\mathscr{T}$.

by the well-known hyperbolic cosine rule, this condition translates into the set of equations

$$
\frac{\cos \alpha_{l}^{j} \cos \alpha_{l}^{j+1}+\cos \gamma_{l}^{j+2}}{\sin \alpha_{l}^{j} \sin \alpha_{l}^{j+1}}=\frac{\cos ^{2} \beta+\cos \beta}{\sin ^{2} \beta}, \quad l=1, \ldots, 2 k, j=1,2,3
$$

The conditions ensuring that the matching exceptional lateral hexagons could be glued by isometries are more involved, and we direct the reader to [Frigerio 2006] for a full discussion of this issue. Under the assumption that equations (2-1) are in force, using the hyperbolic sine rule and [Frigerio 2006, Propositions 1.9, 1.10] one can easily check that these conditions translate into the set of equations

$$
\begin{aligned}
\sin \alpha_{2 i}^{1} \sin \alpha_{2 i-1}^{1} \sin \gamma_{2 i}^{1} \sin \gamma_{2 i-1}^{1}= & \sin \alpha_{2 i}^{2} \sin \alpha_{2 i-1}^{2} \sin \gamma_{2 i}^{2} \sin \gamma_{2 i-1}^{2} \\
= & \sin \alpha_{2 i}^{3} \sin \alpha_{2 i-1}^{3} \sin \gamma_{2 i}^{3} \sin \gamma_{2 i-1}^{3}, \\
& \quad i=1, \ldots, k
\end{aligned}
$$

Consistency around the edges. Since $\gamma_{l}^{1}+\gamma_{l}^{2}+\gamma_{l}^{3}=\pi$ for $l=1, \ldots, 2 k$, the total angle along any half-infinite edge of $\mathscr{T}$ is automatically forced to be equal to $2 \pi$, so consistency around the edges translate into a single equation:

$$
6 \cdot(g-k) \cdot \beta+\sum_{l=1}^{2 k}\left(\alpha_{l}^{1}+\alpha_{l}^{2}+\alpha_{l}^{3}\right)=2 \pi .
$$

Any solution of the consistency equations equations (2-1), (2-2), (2-3) defines a nonsingular hyperbolic structure with geodesic boundary on $M$.

Completeness equations. Let $\left\{\mu_{i}, \lambda_{i}\right\}$, for $i=1, \ldots, k$, be the basis of $H_{1}\left(T_{i} ; \mathbb{Z}\right)$ defined as follows: $\mu_{i}$ is the projection on $T_{i}$ of the edge in the link of the ideal 
vertex of $\Delta_{2 i-1}$ that joins $f_{2 i-1}^{1}$ to $f_{2 i-1}^{2} ; \lambda_{i}$ is the projection on $T_{i}$ of the edge in the link of the ideal vertex of $\Delta_{2 i}$ that joins $f_{2 i}^{3}$ to $f_{2 i}^{2}$. A solution

$$
x=\left(\alpha_{1}^{1}, \alpha_{1}^{2}, \alpha_{1}^{3}, \gamma_{1}^{1}, \gamma_{1}^{2}, \gamma_{1}^{3}, \ldots, \alpha_{2 k}^{1}, \alpha_{2 k}^{2}, \alpha_{2 k}^{3}, \gamma_{2 k}^{1}, \gamma_{2 k}^{2}, \gamma_{2 k}^{3}, \beta\right) \in \mathbb{R}^{12 k+1}
$$

of consistency equations naturally defines an $\operatorname{Aff}(\mathbb{C})$-structure on $T_{i}$ (see [Benedetti and Petronio 1992; Frigerio 2005], for example). We denote by $a_{i}(x) \in \mathbb{C}$ (resp. by $b_{i}(x) \in \mathbb{C}$ ) the dilation component of the holonomy of $\mu_{i}$ (resp. of $\lambda_{i}$ ) corresponding to the $\operatorname{Aff}(\mathbb{C})$-structure defined by $x$ on $T_{i}$. It is well-known that the hyperbolic structure defined by $x$ on $M$ induces a complete metric on the $i$-th end of $M$ if and only if $a_{i}(x)=b_{i}(x)=1$. Moreover, one can explicitly compute $a_{i}$ and $b_{i}$ in terms of the dihedral angles, using for example [Frigerio 2006, Proposition 1.14]:

\section{Theorem 2.2.}

$$
\begin{aligned}
& a_{i}(x)=\left(\left(\sin \gamma_{2 i-1}^{1} \sin \gamma_{2 i}^{2}\right) /\left(\sin \gamma_{2 i-1}^{2} \sin \gamma_{2 i}^{1}\right)\right) \exp \left(i\left(\gamma_{2 i-1}^{3}-\gamma_{2 i}^{3}\right)\right), \\
& b_{i}(x)=\left(\left(\sin \gamma_{2 i-1}^{2} \sin \gamma_{2 i}^{3}\right) /\left(\sin \gamma_{2 i-1}^{3} \sin \gamma_{2 i}^{2}\right)\right) \exp \left(i\left(\gamma_{2 i-1}^{1}-\gamma_{2 i}^{1}\right)\right) .
\end{aligned}
$$

The next theorem shows that a solution of consistency and completeness equations always exists, and is as symmetric as possible.

Theorem 2.3 [Frigerio et al. 2003]. There exist constants $\bar{\alpha}_{g, k}, \bar{\beta}_{g, k} \in(0, \pi / 3)$ such that the point

$$
x_{0}=\left(\bar{\alpha}_{g, k}, \bar{\alpha}_{g, k}, \bar{\alpha}_{g, k}, \pi / 3, \pi / 3, \pi / 3, \ldots, \bar{\alpha}_{g, k}, \bar{\alpha}_{g, k}, \bar{\alpha}_{g, k}, \pi / 3, \pi / 3, \pi / 3, \bar{\beta}_{g, k}\right)
$$

in $\mathbb{R}^{12 k+1}$ provides the unique solution of the consistency and completeness equations for $\mathcal{T}$.

Thus the complete hyperbolic structure of $M$ induces on each boundary torus the regular hexagonal Euclidean structure which is obtained by gluing two Euclidean equilateral triangles.

Dehn filling equations. From now on we denote by $\Omega_{g, k} \subset \mathbb{R}^{12 k+1}$ the set of solutions of consistency equations for $\mathscr{T}$ (it is clear that this set indeed depends only on $g$ and $k$ ). If $x \in \Omega_{g, k}$ is as in equation (2-4), we set

$$
\begin{aligned}
& u_{i}(x)=\ln a_{i}(x)=\ln \left(\left(\sin \gamma_{2 i-1}^{1} \sin \gamma_{2 i}^{2}\right) /\left(\sin \gamma_{2 i-1}^{2} \sin \gamma_{2 i}^{1}\right)\right)+i\left(\gamma_{2 i-1}^{3}-\gamma_{2 i}^{3}\right), \\
& v_{i}(x)=\ln b_{i}(x)=\ln \left(\left(\sin \gamma_{2 i-1}^{2} \sin \gamma_{2 i}^{3}\right) /\left(\sin \gamma_{2 i-1}^{3} \sin \gamma_{2 i}^{2}\right)\right)+i\left(\gamma_{2 i-1}^{1}-\gamma_{2 i}^{1}\right) .
\end{aligned}
$$

It is proved in [Frigerio 2006] that near $x_{0}$, the space $\Omega_{g, k} \subset \mathbb{R}^{12 k+1}$ is a smooth manifold of real dimension $2 k$, whose tangent space $T_{x_{0}} \Omega_{g, k}$ at $x_{0}$ is given by the solutions of the linearization of consistency equations (2-1), (2-2), (2-3). Moreover, there exists a small neighbourhood $U$ of $x_{0}$ in $\Omega_{g, k}$ with the following properties:

(1) For $x \in U$, we have $u_{i}(x)=0 \Leftrightarrow v_{i}(x)=0 \Leftrightarrow$ the metric structure defined by $x$ is complete at the $i$-th end of $M$; 
(2) The map $\left(u_{1}, \ldots, u_{k}\right): U \rightarrow \mathbb{C}^{k}$ is a diffeomorphism between $U$ and an open neighbourhood of 0 in $\mathbb{C}^{k}$.

Let $U$ be a sufficiently small neighbourhood of $x_{0}$ in $\Omega_{g, k}$ and let $x \in U$. For $j=1, \ldots, k$, we define the $j$-Dehn filling coefficient $\left(p_{j}(x), q_{j}(x)\right) \in \mathbb{R}^{2} \cup\{\infty\}$ as follows: if $u_{j}(x)=0$, then $\left(p_{j}(x), q_{j}(x)\right)=\infty$; otherwise, $p_{j}(x), q_{j}(x)$ are the unique real solutions of the equation

$$
p_{j}(x) u_{j}(x)+q_{j}(x) v_{j}(x)=2 \pi i .
$$

The existence and uniqueness of such solutions near $x_{0}$ are proved in [Frigerio 2006]. Set

$$
d=\left(d_{1}, \ldots, d_{k}\right): U \rightarrow \prod_{i=1}^{k} S^{2}, \quad d_{j}(x)=\left(p_{j}(x), q_{j}(x)\right) \in S^{2}=\mathbb{R}^{2} \cup\{\infty\} .
$$

It is proved in the same paper that, if $U$ is small enough, the map $d$ defines a diffeomorphism onto an open neighbourhood of $(\infty, \ldots, \infty)$ in $S^{2} \times \cdots \times S^{2}$.

For $x \in \Omega_{g, k}$ we denote by $M(x)$ the hyperbolic structure induced on $M$ by $x$, and by $\widehat{M}(x)$ the metric completion of $M(x)$. We also define the set

$$
\begin{array}{r}
I \Omega_{g, k}=\left\{x \in U \subset \Omega_{g, k}: \text { for } i=1, \ldots, k \text { the } i\right. \text {-th Dehn filling coefficient } \\
\text { associated to } x \text { is either } \infty \text { or a pair of coprime integers }\} .
\end{array}
$$

Thurston's hyperbolic Dehn filling Theorem (see, for example, [Thurston 1979; Neumann and Zagier 1985; Benedetti and Petronio 1992; Frigerio 2005]) asserts that if $U$ is sufficiently small and $x$ belongs to $I \Omega_{g, k} \cap U$, then $\widehat{M}(x)$ admits a complete finite-volume smooth hyperbolic structure which is obtained by adding to $M(x)$ a closed geodesic at any cusp with noninfinite Dehn filling coefficient. From a topological point of view, $\widehat{M}(x)$ is obtained by filling the $i$-th cusp of $M$ along the slope $p_{i}(x) \mu_{i}+q_{i}(x) \lambda_{i}$ if $\left(p_{i}(x), q_{i}(x)\right) \neq \infty$, and by leaving the $i$-th cusp of $M$ unfilled if $\left(p_{i}(x), q_{i}(x)\right)=\infty, i=1, \ldots, k$.

For $U$ sufficiently small, take $x \in I \Omega_{g, k} \cap U$ and suppose $\left(p_{j}(x), q_{j}(x)\right) \neq \infty$. Let $\ell_{j} \subset \widehat{M}(x)$ be the added geodesic at the $j$-th cusp of $M$ and let $L_{\mathbb{C}}^{x}\left(\ell_{j}\right)$ be its complex length. Choose integers $r_{j}(x), s_{j}(x)$ with $p_{j}(x) s_{j}(x)-q_{j}(x) r_{j}(x)=-1$. It is proved in [Neumann and Zagier 1985] that

$$
L_{\mathbb{C}}^{x}\left(\ell_{j}\right)=r_{j}(x) u_{j}(x)+s_{j}(x) v_{j}(x) .
$$

\section{Isolation of cusps}

We now study small deformations of the complete hyperbolic structure of $M$ by analyzing deformations of the shapes of the geometric tetrahedra of $\mathscr{T}$. For $x \in \Omega_{g, k}$ let $\ell(x) \in \mathbb{R}$ be the length of any boundary edge of any compact lateral hexagon 
in the geometric realization of $\mathscr{T}$ parameterized by $x$. The following results are proved in [Frigerio 2006, Section 3].

Lemma 3.1. The map $\ell: \Omega_{g, k} \rightarrow \mathbb{R}$ is smooth and $d \ell_{x_{0}}=0$.

Lemma 3.2. For $x \in \Omega_{g, k}$ let $\beta(x)=x^{12 k+1}$ be the dihedral angle along the edges of any compact tetrahedron in the geometric realization of $\mathcal{T}$ parameterized by $x$. Then $d \beta_{x_{0}}=0$.

Infinitesimal deformations. We begin by looking for explicit equations for the tangent space $T_{x_{0}} \Omega_{g, k}$. So fix a smooth $\operatorname{arc} \varphi:(-\varepsilon, \varepsilon) \rightarrow \Omega_{g, k}$. For any smooth $f: \Omega_{g, k} \rightarrow \mathbb{R}$, denote by $\dot{f}$ the derivative of $f \circ \varphi$ at $t=0$. With notation as on page 344, if $\theta(t)$ is the geometric realization of $\mathscr{T}$ parameterized by $\varphi(t) \in \Omega_{g, k}$, we set $\alpha_{l}^{j}(t)=\theta(t)\left(e_{l}^{j}\right), \gamma_{l}^{j}(t)=\theta(t)\left(f_{l}^{j}\right)$.

Recall that for every $l=1, \ldots, 2 k, j=1,2,3$ we have

$$
\ell(\varphi(t))=\frac{\cos \alpha_{l}^{j+1}(t) \cdot \cos \alpha_{l}^{j+2}(t)+\cos \gamma_{l}^{j}(t)}{\sin \alpha_{l}^{j+1}(t) \cdot \sin \alpha_{l}^{j+2}(t)},
$$

where apices are considered mod 3. Moreover, by Lemma 3.1 we have $\dot{\ell}=0$, so differentiating at 0 the equation above we easily get

$$
\sqrt{3}\left(\dot{\alpha}_{l}^{j+1}+\dot{\alpha}_{l}^{j+2}\right) \cos \bar{\alpha}_{g, k}+\dot{\gamma}_{l}^{j} \sin \bar{\alpha}_{g, k}=0 .
$$

Summing up these equations for $j=1,2,3$ and observing that $\dot{\gamma}_{l}^{1}+\dot{\gamma}_{l}^{2}+\dot{\gamma}_{l}^{3}=0$ we obtain

$$
\dot{\alpha}_{l}^{1}+\dot{\alpha}_{l}^{2}+\dot{\alpha}_{l}^{3}=0
$$

whence

$$
\sqrt{3} \dot{\alpha}_{l}^{j} \cos \bar{\alpha}_{g, k}=\dot{\gamma}_{l}^{j} \sin \bar{\alpha}_{g, k} .
$$

Let $i \in\{1, \ldots, k\}$. Evaluating equations (2-2) along $\varphi$ and differentiating at 0 we get

$$
\begin{aligned}
\sqrt{3}\left(\dot{\alpha}_{2 i-1}^{1}+\dot{\alpha}_{2 i}^{1}\right) \cos \bar{\alpha}_{g, k}+ & \left(\dot{\gamma}_{2 i-1}^{1}+\dot{\gamma}_{2 i}^{1}\right) \sin \bar{\alpha}_{g, k} \\
& =\sqrt{3}\left(\dot{\alpha}_{2 i-1}^{2}+\dot{\alpha}_{2 i}^{2}\right) \cos \bar{\alpha}_{g, k}+\left(\dot{\gamma}_{2 i-1}^{2}+\dot{\gamma}_{2 i}^{2}\right) \sin \bar{\alpha}_{g, k} \\
& =\sqrt{3}\left(\dot{\alpha}_{2 i-1}^{3}+\dot{\alpha}_{2 i}^{3}\right) \cos \bar{\alpha}_{g, k}+\left(\dot{\gamma}_{2 i-1}^{3}+\dot{\gamma}_{2 i}^{3}\right) \sin \bar{\alpha}_{g, k} .
\end{aligned}
$$

Together with equations (3-1) and (3-2), this implies

$$
\dot{\alpha}_{2 i-1}^{1}=-\dot{\alpha}_{2 i}^{1}, \quad \dot{\alpha}_{2 i-1}^{2}=-\dot{\alpha}_{2 i}^{2}, \quad \dot{\alpha}_{2 i-1}^{3}=-\dot{\alpha}_{2 i}^{3} .
$$


We can now summarize all these computations giving explicit equations for $T_{x_{0}} \Omega_{g, k}$. Let $Z$ be the linear subspace of $\mathbb{R}^{12}$ defined by the equations

$$
\begin{aligned}
& \left(\sqrt{3} \cos \bar{\alpha}_{g, k}\right) x_{1}=\left(\sin \bar{\alpha}_{g, k}\right) x_{4}, \\
& \left(\sqrt{3} \cos \bar{\alpha}_{g, k}\right) x_{2}=\left(\sin \bar{\alpha}_{g, k}\right) x_{5}, \\
& \left(\sqrt{3} \cos \bar{\alpha}_{g, k}\right) x_{3}=\left(\sin \bar{\alpha}_{g, k}\right) x_{6}, \\
& \left(\sqrt{3} \cos \bar{\alpha}_{g, k}\right) x_{7}=\left(\sin \bar{\alpha}_{g, k}\right) x_{10}, \\
& \left(\sqrt{3} \cos \bar{\alpha}_{g, k}\right) x_{8}=\left(\sin \bar{\alpha}_{g, k}\right) x_{11}, \\
& \left(\sqrt{3} \cos \bar{\alpha}_{g, k}\right) x_{9}=\left(\sin \bar{\alpha}_{g, k}\right) x_{12}, \\
& x_{1}+x_{7}=x_{2}+x_{8}=x_{3}+x_{9}=0, \\
& x_{1}+x_{2}+x_{3}=0 .
\end{aligned}
$$

Observe that $\operatorname{dim}_{\mathbb{R}} Z=2$. For $i=1, \ldots, k$ let $r_{i}: \mathbb{R}^{12 k+1} \rightarrow \mathbb{R}^{12}$ be the map defined by $r_{i}(x)=\left(x_{12 i-11}, x_{12 i-10}, \ldots, x_{12 i-1}, x_{12 i}\right)$. Let

$$
\bar{Z}=\left\{x \in \mathbb{R}^{12 k+1}: x_{12 k+1}=0, r_{i}(x) \in Z \text { for } i=1, \ldots, k\right\}
$$

be the product of one copy of $Z$ for each cusp. Now $\operatorname{dim}_{\mathbb{R}} \bar{Z}=2 k=\operatorname{dim}_{\mathbb{R}} T_{x_{0}} \Omega_{g, k}$, and by Lemma 3.2 and equations (3-1), (3-2), (3-3) we also have $T_{x_{0}} \Omega_{g, k} \subseteq \bar{Z}$, whence:

Proposition 3.3. $T_{x_{0}} \Omega_{g, k}=\bar{Z}$.

Isolation of cusps. We now go into the proof of Theorem 1.3. Let $C_{1}, \ldots, C_{k}$ be the cusps of our fixed manifold $M \in M_{g, k}$ corresponding to the boundary tori $T_{1}, \ldots, T_{k}$. We look for equations defining the set of structures in $\Omega_{g, k}$ which are complete at $C_{1}, \ldots, C_{h}$. To this aim we set:

$$
J_{h}=\left\{x \in \mathbb{R}^{12 k+1}: x_{12 i+1}=x_{12 i+2}=x_{12 i+3} \text { for all } i=0, \ldots, h-1\right\} .
$$

It is easily seen that $T_{x_{0}} J_{h}+T_{x_{0}} \Omega_{g, k}=J_{h}+\bar{Z}=\mathbb{R}^{12 k+1}=T_{x_{0}} \mathbb{R}^{12 k+1}$, so basic results about transverse intersections of submanifolds readily imply the following:

Lemma 3.4. Near $x_{0}$, the set $J_{h} \cap \Omega_{g, k}$ is a smooth submanifold of $\Omega_{g, k}$ of real dimension $2(k-h)$.

Let $\Delta$ be a topological partially truncated tetrahedron with ideal vertex $v_{0}$, and take $\vartheta \in(0, \pi / 3)$. Then there exists, up to isometry, exactly one geometric realization of $\Delta$ with dihedral angles $\pi / 3$ along the internal edges emanating from $v_{0}$, and angle $\vartheta$ along the other internal edges. We denote this geometric tetrahedron by $\Delta^{\vartheta}$. 
Proposition 3.5. For each $l=1, \ldots, 2 h$ let $\Delta_{l}^{*}(p)$ be the geometric realization of $\Delta_{l}$ parameterized by $p \in J_{h} \cap \Omega_{g, k}$. Then a real number $\vartheta(p) \in(0, \pi / 3)$ exists such that $\Delta_{l}^{*}(p)$ is isometric to $\Delta^{\vartheta(p)}$ for $l=1, \ldots, 2 h$.

Proof. For $l=1, \ldots, 2 h, j=1,2,3$ let $T_{l}^{j}(p)$ be the truncation triangle of $\Delta_{l}^{*}(p)$ having a vertex on the edge $f_{l}^{j}$. Fix $i \in\{0, \ldots, h-1\}$, consider the tetrahedron $\Delta_{2 i+1}^{*}(p)$, and let $H$ be the compact lateral hexagon of $\Delta_{2 i+1}^{*}(p)$. Since the internal edges of $H$ have all the same length, by an easy result in hyperbolic trigonometry also the boundary edges of $H$ have all the same length. Together with the equality $x_{12 i+1}(p)=x_{12 i+2}(p)=x_{12 i+3}(p)$ (which holds by hypothesis, since $\left.p \in J_{h}\right)$, this implies that the triangles $T_{2 i+1}^{1}(p), T_{2 i+1}^{2}(p)$ and $T_{2 i+1}^{3}(p)$ are isosceles and isometric to each other. This gives in turn $x_{12 i+4}(p)=x_{12 i+5}(p)=x_{12 i+6}(p)$, so $\Delta_{2 i+1}^{*}$ is isometric to $\Delta^{\xi_{2 i+1}}$ for some $\xi_{2 i+1}=x_{12 i+1}(p) \in(0, \pi / 3)$. Moreover, since the noncompact faces of $\Delta_{2 i+1}^{*}$ are isometrically glued to the noncompact faces of $\Delta_{2 i+2}^{*}$ we easily see that the truncation triangles $T_{2 i+2}^{1}(p), T_{2 i+2}^{2}(p)$ and $T_{2 i+2}^{3}(p)$ are isosceles and isometric to each other. This forces $x_{12 i+7}(p)=x_{12 i+8}(p)=$ $x_{12 i+9}(p)=\xi_{2 i+2}$ and $x_{12 i+10}(p)=x_{12 i+11}(p)=x_{12 i+12}(p)=\pi / 3$, so $\Delta_{2 i+2}^{*}$ is isometric to $\Delta^{\xi_{2 i+2}}$ for some $\xi_{2 i+2} \in(0, \pi / 3)$.

Finally, since the length of the compact internal edges of the $\Delta_{l}^{*}$ 's does not depend on $l$, we have $\xi_{1}=\cdots=\xi_{2 h}$, whence the conclusion.

Corollary 3.6. Let $p$ be a point in $J_{h} \cap \Omega_{g, k}$ and denote by $M(p)$ the hyperbolic structure defined by $p$ on $M$. Then for all $i=1, \ldots, h$ the following holds:

- $M(p)$ induces a complete metric on the cusp $C_{i}$;

- The Euclidean structure induced on $T_{i}$ by $M(p)$ is isometric to the regular hexagonal structure induced on $T_{i}$ by the complete hyperbolic structure $M\left(x_{0}\right)$.

The corollary just stated says that the Euclidean structures on $T_{1}, \ldots, T_{h}$ are not affected by the deformations of the hyperbolic metric on $M$ which correspond to points in $J_{h} \cap \Omega_{g, k}$. Therefore to conclude the proof of Theorem 1.3 we only need the following:

Proposition 3.7. Let $K_{h}$ be the subset of $\Omega_{g, k}$ corresponding to the structures inducing complete metrics on $C_{1}, \ldots, C_{h}$. Then there exists a neighbourhood $V$ of $x_{0}$ in $\Omega_{g, k}$ with $K_{h} \cap V=J_{h} \cap V$.

Proof. By Lemma 3.4 there exists a neighbourhood $W$ of $x_{0}$ in $\Omega_{g, k}$ such that both $K_{h} \cap W$ and $J_{h} \cap W$ are smooth submanifolds of $\Omega_{g, k}$ of real dimension $2(k-h)$. Moreover Corollary 3.6 shows that $J_{h} \cap W \subset K_{h} \cap W$, whence $J_{h} \cap V=K_{h} \cap V$ for some (maybe smaller) neighbourhood $V$ of $x_{0}$ in $\Omega_{g, k}$. 
Nonisolation of the boundary. We now sketch the proof of Theorem 1.4. Let Teich $(\partial M)$ be the Teichmüller space of hyperbolic structures on $\partial M, i$. e. the space of equivalence classes of hyperbolic metrics on $\partial M$, where two such metrics are considered equivalent if they are isometric through a diffeomorphism homotopic to the identity of $\partial M$. For $x \in \Omega_{g, k}$ we denote by $M(x)$ the hyperbolic structure defined on $M$ by $x$, and by $B(x) \in \operatorname{Teich}(\partial M)$ the equivalence class of the hyperbolic structure induced by $M(x)$ on $\partial M$. Given a nontrivial element $\gamma \in \pi_{1}(\partial M)$, we also denote by $L_{\gamma}(x)$ the length, with respect to the hyperbolic structure $B(x) \in \operatorname{Teich}(\partial M)$, of the unique geodesic representative of $\gamma$. It is wellknown that Teich $(\partial M)$ admits a structure of differentiable manifold such that the maps $B: \Omega_{g, k} \rightarrow \operatorname{Teich}(\partial M)$ and $L_{\gamma}: \Omega_{g, k} \rightarrow \mathbb{R}$ are smooth.

It is proved in [Frigerio 2006] that $d B_{x_{0}}=0$, thus in order to prove that the geodesic boundary of $M$ is not isolated from the cusps we need to analyze the map $B$ up to the second order in a neighbourhood of $x_{0}$. The key result is this:

Proposition 3.8. There exist a smooth path $\bar{\rho}:(-\varepsilon, \varepsilon) \rightarrow \Omega_{g, k}$ and an element $\bar{\gamma} \in \pi_{1}(\partial M)$ such that $\bar{\rho}(0)=x_{0}$ and the map $t \mapsto L_{\bar{\gamma}}(\bar{\rho}(t))$ has nonzero second derivative at 0 .

In order to find the suitable $\bar{\rho}$ and $\bar{\gamma}$, one has to explicitly describe the combinatorics of the triangulation of $\partial M$ induced by the triangulation of $M$, and to compute the second derivatives of consistency equations at $x_{0}$. Since computations are quite long, we address the reader to [Frigerio 2005] for a complete proof of Proposition 3.8. In the rest of this section we show how Theorem 1.4 can be deduced from Proposition 3.8 .

Definition 3.9. Let $y_{0}$ be a point of a smooth $n$-manifold $Y$ and let $\varphi: U \rightarrow \mathbb{R}^{n}$ be a diffeomorphism with $\varphi\left(y_{0}\right)=0$, where $U \subset Y$ is a small open neighbourhood of $y_{0}$. Let $0 \neq v \in T_{y_{0}} Y$, and consider a sequence $\left\{y_{j}\right\}_{j \in \mathbb{N}} \subset U \backslash\left\{y_{0}\right\}$. We say that $y_{n}$ converges to $y_{0}$ along $v$ if

$$
\lim _{j \rightarrow \infty} y_{j}=y_{0}, \quad \lim _{j \rightarrow \infty} \frac{\varphi\left(y_{j}\right)}{\left\|\varphi\left(y_{j}\right)\right\|}=\frac{d \varphi_{y_{0}}(v)}{\left\|d \varphi_{y_{0}}(v)\right\|},
$$

where we are identifying $T_{0} \mathbb{R}^{n}$ with $\mathbb{R}^{n}$, endowed with the Euclidean norm $\|\cdot\|$.

Recall the set $I \Omega_{g, k} \subset \Omega_{g, k}$ defined in Equation (2-5), and let $\left\{y_{n}\right\}_{n \in \mathbb{N}} \subset I \Omega_{g, k} \backslash$ $\left\{x_{0}\right\}$ be a sequence converging to $x_{0}$ along $\dot{\bar{\rho}}(0)$. By construction, up to passing to a subsequence we have $B\left(y_{n}\right) \neq B\left(x_{0}\right)$ for every $n \in \mathbb{N}$.

Since $y_{i} \in I \Omega_{g, k}$, the metric completion of the structure induced on $M$ by $y_{i}$ gives a nonsingular hyperbolic 3-manifold $N_{i}$. Recall that the mapping class group $M \mathscr{C} \mathscr{G}(\partial M)$ of $\partial M$ acts properly discontinuously on Teich $(\partial M)$, so there exists a neighbourhood $W$ of $B\left(x_{0}\right)$ in Teich $(\partial M)$ such that the set $\{\psi \in M \mathscr{C} \mathscr{G}(\partial M)$ : $\psi(W) \cap W \neq \varnothing\}$ is finite. Up to passing to a subsequence, we may suppose that 
the equivalence classes of the $\partial N_{j}$ 's are pairwise distinct as elements in Teich $(\partial M)$, and that $\partial N_{i} \in U$ for all $i \in \mathbb{N}$. This readily implies that among the $\partial N_{j}$ 's there are infinitely many pairwise nonisometric hyperbolic surfaces, whence Theorem 1.4.

\section{Similar fillings}

Kojima [1990] proved that every complete finite-volume hyperbolic manifold $N$ with nonempty geodesic boundary admits a canonical decomposition into geometric polyhedra. Such a decomposition is in a certain sense dual to the cut-locus of the boundary of $N$, and is therefore unique. As a corollary, this implies the fact (that we will be using later) that the isometry group of $N$ is canonically isomorphic to the group of combinatorial automorphisms of the Kojima decomposition of $N$.

Proposition 4.1. Any shortest return path in $N$ is an edge of the Kojima decomposition of N. Moreover, any compact regular partially truncated tetrahedron isometrically immersed in $N$ whose internal edges are shortest return paths is a piece of the canonical decomposition of $N$.

Theorem 4.2 [Frigerio et al. 2003]. Let $M \in \mathcal{M}_{g, k}$ with $\partial M=\Sigma_{g} \sqcup\left(\bigsqcup_{i=1}^{k} T_{i}\right)$.

(1) $M$ has a unique triangulation with $g+k$ tetrahedra, which gives the canonical Kojima decomposition of $M$.

(2) The volume of the complete hyperbolic structure of $M$ depends only on $g$ and $k$.

(3) The Heegaard genus of $\left(M, \Sigma_{g}, \bigsqcup_{i=1}^{k} T_{i}\right)$ is $g+1$.

(4) $H_{1}(M ; \mathbb{Z})=\mathbb{Z}^{g+k}$.

(5) The Turaev-Viro invariant $\operatorname{TV}_{r}(M)$ depends only on $r, g$ and $k$.

The next result shows that manifolds in $M_{g, k}$ are geometrically similar to each other:

Theorem 4.3. Let $M \in \mathcal{M}_{g, k}$ be endowed with its complete hyperbolic structure.

(1) The cusp volume of $M$ depends only on $g$ and $k$;

(2) The Euclidean structures on the boundary tori of $M$ are all isometric to the regular hexagonal one;

(3) The length of the shortest return path of $M$ depends only on $g$ and $k$.

Proof. Manipulating equation (2-1), one can easily prove that $\bar{\alpha}_{g, k}<\bar{\beta}_{g, k}<2 \bar{\alpha}_{g, k} \leqslant$ $\pi / 3$. Together with some computations in hyperbolic space, this implies that each noncompact tetrahedron of the Kojima decomposition of $M$ contains a horocusp neighbourhood of its ideal vertex which is tangent to the truncation triangles. Thus a maximal regular horocusp neighbourhood for $M$ is obtained by gluing the maximal horocusp neighbourhoods of the ideal vertices of the noncompact tetrahedra 
of the Kojima decomposition, whence point (1). Point (2) has already been established and point (3) is a consequence of Proposition 4.1 and Theorem 4.2-(1).

The following result refines Theorem 4.2-(4), and can be proved just by looking at the description of the combinatorics of the Kojima decomposition of $M$ given in Proposition 1.1 (see [Frigerio 2005] for a complete proof).

Proposition 4.4. Let $M \in M_{g, k}$. Then we have the exact sequence

$$
0 \longrightarrow H_{1}\left(\bigsqcup_{i=1}^{k} T_{i} ; \mathbb{Z}\right) \stackrel{i_{*}}{\longrightarrow} H_{1}(M ; \mathbb{Z}) \longrightarrow \mathbb{Z}^{g-k} \longrightarrow 0,
$$

where $i_{*}$ is the map induced by the inclusion $i: \bigsqcup_{i=1}^{k} T_{i} \rightarrow M$.

4A. Boundary slopes and Dehn filling. Let $T_{i}$ be the $i$-th boundary torus of a manifold $M \in M_{g, k}$, and recall that the unique complete finite-volume hyperbolic structure on $M$ induces on $T_{i}$ a Euclidean structure defined up to similarity. For the sake of simplicity, we endow $T_{i}$ with a fixed Euclidean structure choosing the scale factor in such a way that $T_{i} \cong \mathbb{C} / \Gamma$, where $\Gamma$ is the discrete additive subgroup of $\mathbb{C}$ with generators $1, e^{i \pi / 3}$. We denote by $\mathcal{M}\left(T_{i}\right)$ the group of isotopy classes of isometries of $T_{i}$. Of course $\mathcal{M}\left(T_{i}\right)$ acts on the set of slopes on $T_{i}$.

Let $D_{6}$ be the dihedral group with 12 elements, $i$. e. the group of isometries of $\mathbb{C}$ generated by the rotation $r: \mathbb{C} \rightarrow \mathbb{C}, r(z)=e^{i \pi / 3} \cdot z$ and the reflection $s: \mathbb{C} \rightarrow \mathbb{C}, s(z)=\bar{z}$. Any element of $D_{6}$ induces an isometry of $T_{i}$, and any isometry of $T_{i}$ lifts up to isotopy to an element of $D_{6}$. Thus $\mathcal{M}\left(T_{i}\right)$ is canonically isomorphic to $D_{6}$.

Let $\mu_{i}, \lambda_{i}$ be the preferred basis of $H_{1}\left(T_{i} ; \mathbb{Z}\right.$ ) chosen in Section 2 (see bottom of page 345). In what follows we will often represent slopes as indivisible elements in $H_{1}\left(T_{i} ; \mathbb{Z}\right)$ without emphasizing the fact that each slope corresponds in fact to two such elements. Any slope $s$ on $T_{i}$ determines a well-defined isotopy class of geodesics on $T_{i}$, and we denote by $L(s)$ the Euclidean length of such geodesics. An elementary calculation shows that if $s=p \cdot \mu_{i}+q \cdot \lambda_{i}$, then $L(s)=\sqrt{p^{2}+q^{2}-p q}$. Thus, if $\left\{\kappa_{1}<\kappa_{2}<\cdots<\kappa_{n}<\cdots\right\}$ is the set of lengths of slopes on $T_{i}$, then the following holds:

- There are exactly three slopes of length $\kappa_{1}=1$. They are represented by $\mu_{i}, \lambda_{i}$ and $\mu_{i}+\lambda_{i}$, and they are $M\left(T_{i}\right)$-equivalent to each other.

- There are exactly three slopes of length $\kappa_{2}=\sqrt{3}$. They are represented by $\mu_{i}-\lambda_{i}, \mu_{i}+2 \cdot \lambda_{i}$ and $2 \cdot \mu_{i}+\lambda_{i}$, and they are $M\left(T_{i}\right)$-equivalent to each other.

- If $s$ is a slope with $L(s) \geqslant \kappa_{3}=\sqrt{7}$, then there exist exactly six slopes $M\left(T_{i}\right)$ equivalent to $s$.

The following result completely classifies the Dehn fillings of elements in $\mu_{g, k}$. 
Theorem 4.5 [Frigerio et al. 2003]. Let $M \in M_{g, k}$ with $\partial M=\Sigma_{g} \sqcup\left(\bigsqcup_{i=1}^{k} T_{i}\right)$, let $h \leqslant k$, let $s_{i}$ be a slope on $T_{i}$ for $i=1, \ldots, h$ and $N=M\left(s_{1}, \ldots, s_{h}\right)$. Then $N$ is hyperbolic if and only if $L\left(s_{i}\right) \geqslant \kappa_{3}$ for all $i=1, \ldots, h$. Moreover, when $N$ is hyperbolic the Heegaard genus of $\left(N, \Sigma_{g}, T_{h+1} \sqcup \cdots \sqcup T_{k}\right)$ is $g+1$.

4B. Symmetries of $\boldsymbol{\Omega}_{\boldsymbol{g}, \boldsymbol{k}}$. Let Aut $\boldsymbol{\Omega}_{g, k}$ denote the set of diffeomorphisms of $\Omega_{g, k}$ onto itself. We describe some elements in Aut $\Omega_{g, k}$ and explain how they act on the space of Dehn filling coefficients. In order to clarify our arguments it is convenient to denote the coordinates of $\mathbb{R}^{12 k+1}$ as in equation (2-4):

$$
\beta(x)=x_{12 k+1}, \quad \alpha_{l}^{j}(x)=x_{6(l-1)+j}, \quad \gamma_{l}^{j}(x)=x_{6(l-1)+3+j},
$$

for $l=1, \ldots, 2 k$ and $j=1,2,3$.

Let $x \in \Omega_{g, k}$, fix $i \in\{1, \ldots, k\}$ and take an element $\sigma$ of the symmetric group $\mathfrak{S}_{3}$. Define $x^{\prime} \in \mathbb{R}^{12 k+1}$ by

$$
\begin{aligned}
& \alpha_{l}^{j}\left(x^{\prime}\right)=\alpha_{l}^{\sigma^{-1}(j)}(x), \quad \gamma_{l}^{j}\left(x^{\prime}\right)=\gamma_{l}^{\sigma^{-1}(j)}(x) \quad \text { if } l=2 i-1,2 i ; \\
& \alpha_{l}^{j}\left(x^{\prime}\right)=\alpha_{l}^{j}(x), \quad \gamma_{l}^{j}\left(x^{\prime}\right)=\gamma_{l}^{j}(x) \quad \text { if } l \neq 2 i-1,2 i \text {; } \\
& \beta\left(x^{\prime}\right)=\beta(x) \text {, }
\end{aligned}
$$

Due to the symmetry of consistency equations, $x^{\prime}$ lies in $\Omega_{g, k}$. Roughly speaking, this means that we can make $\sigma$ act on the $i$-th cusp, while leaving all the other cusps and the compact tetrahedra unchanged, thus defining an element $\widehat{\sigma}_{i} \in$ Aut $\Omega_{g, k}$.

Another symmetry $\zeta_{i}: \Omega_{g, k} \rightarrow \Omega_{g, k}$ exists which corresponds to interchanging the rôles of the tetrahedra $\Delta_{2 i-1}$ and $\Delta_{2 i}, i$. e. to swapping the indices of the angles of $\Delta_{2 i}, \Delta_{2 i-1}$, while leaving the shape of all the other tetrahedra unchanged. It is easily seen that the map

$$
\varphi_{i}: \mathfrak{S}_{3} \times \mathbb{Z} / 2 \rightarrow \text { Aut } \Omega_{g, k}, \quad \varphi_{i}(\sigma, \varepsilon)=\widehat{\sigma}_{i} \circ \zeta_{i}^{\varepsilon}, \quad \sigma \in \mathfrak{S}_{3}, \varepsilon=0,1
$$

is an injective homomorphism whose image is a certain subgroup $\operatorname{Sym}_{i} \Omega_{g, k}$ of Aut $\Omega_{g, k}$.

Again because of the symmetries of consistency equations, we can also permute arbitrarily the shapes of the cusps: in this way any element $\kappa$ of the symmetric group $\mathfrak{S}_{k}$ induces a well-defined symmetry $\tilde{\kappa} \in$ Aut $\Omega_{g, k}$. The map $\kappa \mapsto \tilde{\kappa}$ defines an injective homomorphism $v: \mathfrak{S}_{k} \rightarrow$ Aut $\Omega_{g, k}$.

Denote by $\operatorname{Sym} \Omega_{g, k}$ the subgroup of the group Aut $\Omega_{g, k}$ generated by the union $v\left(\mathfrak{S}_{k}\right) \cup\left(\bigcup_{i=1}^{k} \operatorname{Sym}_{i} \Omega_{g, k}\right)$. Since elements in $\operatorname{Sym}_{i} \Omega_{g, k}$ commute with elements in $\operatorname{Sym}_{j} \Omega_{g, k}$ whenever $i \neq j$, the kernel of the natural epimorphism $\pi: \operatorname{Sym} \Omega_{g, k} \rightarrow$ $\mathfrak{S}_{k}$ is given by the direct product $\prod_{i=1}^{k} \operatorname{Sym}_{i} \Omega_{g, k}$. Thus

$$
\operatorname{Sym} \Omega_{g, k}=v\left(\mathfrak{S}_{k}\right) \ltimes\left(\prod_{i=1}^{k} \operatorname{Sym}_{i} \Omega_{g, k}\right) \cong \mathfrak{S}_{k} \ltimes\left(\prod_{i=1}^{k} \mathfrak{S}_{3} \times \mathbb{Z} / 2\right) .
$$


Our next task is to investigate how symmetries in $\operatorname{Sym} \Omega_{g, k}$ act on the space of Dehn filling coefficients parameterizing a small neighbourhood of $x_{0}$ in $\Omega_{g, k}$.

4C. Action on Dehn filling coefficients. Denote by $M\left(T_{1} \sqcup \cdots \sqcup T_{k}\right)$ the group of isotopy classes of isometries of $T_{1} \sqcup \cdots \sqcup T_{k}$. For $\sigma \in \mathfrak{S}_{k}$ we define an element $\eta(\sigma) \in \mathcal{M}\left(T_{1} \sqcup \cdots \sqcup T_{k}\right)$ which permutes the marked tori $T_{1}, \ldots, T_{k}$ according to $\sigma$. Namely, $\eta(\sigma)$ is the isotopy class of any element $\sigma^{\prime} \in \operatorname{Isom}\left(T_{1} \sqcup \cdots \sqcup T_{k}\right)$ taking $T_{j}$ to $T_{\sigma(j)}, \mu_{j}$ to $\mu_{\sigma(j)}, \lambda_{j}$ to $\lambda_{\sigma(j)}$ for $j=1, \ldots, k$. The kernel of the natural epimorphism $\pi^{\prime}: \mathcal{M}\left(T_{1} \sqcup \cdots \sqcup T_{k}\right) \rightarrow \mathfrak{S}_{k}$ is canonically isomorphic to $M\left(T_{1}\right) \times \cdots \times M\left(T_{k}\right)$. Therefore

$$
\mu\left(T_{1} \sqcup \cdots \sqcup T_{k}\right)=\eta\left(\mathfrak{S}_{k}\right) \ltimes\left(\prod_{i=1}^{k} \mu\left(T_{i}\right)\right) \cong \mathfrak{S}_{k} \ltimes\left(\prod_{i=1}^{k} D_{6}\right),
$$

where $D_{6} \cong \mathfrak{S}_{3} \times \mathbb{Z} / 2$ is the dihedral group with 12 elements.

From now on we fix our attention on a small neighbourhood $V$ of $x_{0}$ in $\Omega_{g, k}$ such that for all $x \in V$ the Dehn filling coefficient $\left(p_{j}(x), q_{j}(x)\right) \in S^{2}=\mathbb{R}^{2} \cup\{\infty\}$ is well-defined, and the map

$$
d=\left(d_{1}, \ldots, d_{k}\right): V \rightarrow \prod_{i=1}^{k} S^{2}, \quad d_{j}(x)=\left(p_{j}(x), q_{j}(x)\right) \in S^{2}
$$

is a diffeomorphism onto an open neighbourhood of $\{\infty\} \times \cdots \times\{\infty\}$ in the range. It is easily seen that we can also assume $\psi(V)=V$ for all $\psi \in \operatorname{Sym} \Omega_{g, k}$.

Every element $h \in \mathcal{M}\left(T_{1} \sqcup \cdots \sqcup T_{k}\right)$ induces an automorphism of $\bigoplus_{i=1}^{k} H_{1}\left(T_{i} ; \mathbb{R}\right)$. The basis $\mu_{i}, \lambda_{i}$ defines a canonical isomorphism $H_{1}\left(T_{i} ; \mathbb{R}\right) \cong \mathbb{R}^{2}$, so $h$ induces an automorphism $h_{*}$ of $\prod_{i=1}^{k} S^{2}$ that preserves $\{\infty\} \times \cdots \times\{\infty\}$. Looking at how the maps $d_{j}: V \rightarrow S^{2}, i=1, \ldots, k$ change under precompositions with elements in $\operatorname{Sym} \Omega_{g, k}$, after some easy computations [Frigerio 2005], one gets:

Proposition 4.6. For any $\psi \in \operatorname{Sym} \Omega_{g, k}$ there is a unique $h(\psi) \in \mathcal{M}\left(T_{1} \sqcup \cdots \sqcup T_{k}\right)$ such that $d(\psi(x))=h(\psi)_{*}(d(x))$ for all $x \in V$. Moreover the map

$$
\operatorname{Sym} \Omega_{g, k} \rightarrow \mathcal{M}\left(T_{1} \sqcup \cdots \sqcup T_{k}\right), \quad \psi \mapsto h(\psi)
$$

is a group isomorphism which preserves decompositions (4-1), (4-2).

4D. Return paths. We are now interested in describing the shortest return paths in small deformations of the complete structure on $M$. Recall that for $x \in \Omega_{g, k}$ we denote by $M(x)$ the hyperbolic structure induced on $M$ by $x$, and by $\widehat{M}(x)$ the metric completion of $M(x)$. Moreover, if $x \in I \Omega_{g, k}$ then $\widehat{M}(x)$ is a complete finite-volume hyperbolic manifold with geodesic boundary. In this case the unique compact edge in the geometric triangulation of $M(x)$ defines a return path $l_{x}$ in $\widehat{M}(x)$. For $y \in I \Omega_{g, k}$ we denote by $L^{y}$ the length with respect to the hyperbolic metric on $\widehat{M}(y)$. Of course we have $\lim _{y \in I \Omega_{g, k}, y \rightarrow x_{0}} L^{y}\left(l_{y}\right)=L^{x_{0}}\left(l_{x_{0}}\right)$. 
The next lemma can be thought as an extension of the first statement of Proposition 4.1 to manifolds obtained by Dehn filling $M$ along sufficiently long slopes.

Lemma 4.7. There exists a neighbourhood $V^{\prime} \subset V$ of $x_{0}$ in $\Omega_{g, k}$ such that if $x \in I \Omega_{g, k} \cap V^{\prime}$, then $l_{x}$ is the unique shortest return path in $\widehat{M}(x)$.

Proof. We define three real numbers $\mu_{g, k}, \nu_{g, k}, \eta_{g, k}$ as follows: $\mu_{g, k}$ is the distance between a truncation triangle and the opposite lateral hexagon in a noncompact tetrahedron of the canonical decomposition of $M=M\left(x_{0}\right) ; v_{g, k}$ is the distance between a truncation triangle and the opposite lateral hexagon in a compact tetrahedron of the canonical decomposition of $M ; \eta_{g, k}=L^{x_{0}}\left(l_{x_{0}}\right)$ is the length of the compact edge of the canonical decomposition of $M$. A long but straightforward computation [Frigerio 2005, Lemma 3.7.3] shows that $2 \mu_{g, k}>\eta_{g, k}, 2 v_{g, k}>\eta_{g, k}$.

We suppose by contradiction that there exists a sequence $\left\{y_{n}\right\}_{n \in \mathbb{N}} \subset I \Omega_{g, k}$ converging to $x_{0}$ with the following property: a return path $l_{n}^{\prime}$ in $\widehat{M}\left(y_{n}\right)$ exists such that $l_{n}^{\prime} \neq l_{y_{n}}$ for any $n \in \mathbb{N}$ and $\lim \sup _{n \rightarrow \infty} L^{y_{n}}\left(l_{n}^{\prime}\right) \leqslant \eta_{g, k}$. Since the distance between the added geodesics $\widehat{M}(y) \backslash M(y)$ and the geodesic boundary of $M(y)$ approaches $\infty$ as $y$ tends to $x_{0}$, we can suppose $l_{n}^{\prime} \subset M\left(y_{n}\right)$ for any $n \in \mathbb{N}$. Let now $p_{n}, q_{n}$ be the endpoints of $l_{n}^{\prime}$ and $\Delta^{p_{n}}, \Delta^{q_{n}}$ the geometric tetrahedra containing $p_{n}, q_{n}$ respectively. We denote by $l_{p_{n}}^{\prime}$ (resp. $l_{q_{n}}^{\prime}$ ) the connected component of $l_{n}^{\prime} \cap \Delta^{p_{n}}$ (resp. $\left.l_{n}^{\prime} \cap \Delta^{q_{n}}\right)$ containing $p_{n}\left(\right.$ resp. $\left.q_{n}\right)$. Since $l_{n}^{\prime} \neq l_{y_{n}}$ and $l_{n}^{\prime}$ intersect $\partial M\left(y_{n}\right)$ at right angles we easily get

$$
\begin{aligned}
\limsup _{n \rightarrow \infty} L^{y_{n}}\left(l_{n}^{\prime}\right) & \geqslant \liminf _{n \rightarrow \infty} L^{y_{n}}\left(l_{n}^{\prime}\right) \geqslant \liminf _{n \rightarrow \infty} L^{y_{n}}\left(l_{p_{n}}^{\prime}\right)+\liminf _{n \rightarrow \infty} L^{y_{n}}\left(l_{q_{n}}^{\prime}\right) \\
& \geqslant 2 \min \left\{\mu_{g, k}, v_{g, k}\right\}>\eta_{g, k},
\end{aligned}
$$

a contradiction.

4E. Similar fillings. A set of slopes for a complete finite-volume hyperbolic 3manifold $N$ is a set $\mathscr{S}=\left\{s_{i_{1}}, \ldots, s_{i_{h}}\right\}$ of either 0 or 1 slope per boundary torus. If $\mathscr{S}=\left\{s_{i_{1}}, \ldots, s_{i_{h}}\right\}$ is a set of slopes for $N$ we denote by $N(\mathscr{S})$ the manifold obtained by filling $N$ along $s_{i_{1}}, \ldots, s_{i_{h}}$.

Let $M$ and $M^{\prime}$ be elements in $M_{g, k}$ (we do not exclude the case $M=M^{\prime}$ ) with boundary tori $T_{1}, \ldots, T_{k}$ and $T_{1}^{\prime}, \ldots, T_{k}^{\prime}$. We endow each of these tori with the Euclidean metric described in Section 4A above. We say that a set of slopes $\mathscr{P}$ for $M$ is equivalent to the set of slopes $\mathscr{S}^{\prime}$ for $M^{\prime}$ if there exists an orientationpreserving isometry $\psi: T_{1} \sqcup \cdots \sqcup T_{k} \rightarrow T_{1}^{\prime} \sqcup \cdots \sqcup T_{k}^{\prime}$ taking $\mathscr{S}$ onto $\mathscr{Y}^{\prime}$.

Theorem 4.8. Let $M, M^{\prime}$ be elements of $\mathcal{M}_{g, k}$ and $\mathscr{S}$ (resp. $\left.\mathscr{S}^{\prime}\right)$ be a set of slopes for $M\left(\right.$ resp. $\left.M^{\prime}\right)$. Then there exists a positive constant $C$ such that, if all the slopes of $\mathscr{S}$ are longer than $C$ and $\mathscr{S}$ is equivalent to $\mathscr{S}^{\prime}$, then $M(\mathscr{S})$ is geometrically similar to $M^{\prime}\left(\mathscr{S}^{\prime}\right)$. 
Proof. Let $V^{\prime}$ be a neighbourhood of $x_{0}$ in $\Omega_{g, k}$ as in the statement of Lemma 4.7 and $d: V^{\prime} \rightarrow S^{2} \times \cdots \times S^{2}$ be the map defined in equation (4-3). We can choose a positive constant $C$ depending only on $g$ and $k$ such that the following holds: if $\mathscr{Y}=\left\{s_{i_{1}}, \ldots, s_{i_{h}}\right\}$ is a set of slopes for $M$ with $L\left(s_{i_{l}}\right)>C$ for $l=1, \ldots, h$, then any $k$-uple of Dehn filling coefficients corresponding to $\mathscr{S}$ lies in $d\left(V^{\prime}\right)$ (due to the choice of the signs, there exist exactly $2^{h}$ such $k$-uples).

Let now $\mathscr{S}$ be a set of slopes for $M$ whose elements are longer than $C$ and let $\mathscr{S}^{\prime}$ be a set of slopes for $M^{\prime}$ which is equivalent to $\mathscr{Y}$. Choose also points $x, x^{\prime} \in V^{\prime} \subset \Omega_{g, k}$ such that $d(x)$ (resp. $d\left(x^{\prime}\right)$ ) gives a $k$-uple of Dehn filling coefficients corresponding to $\mathscr{S}$ (resp. $\mathscr{S}^{\prime}$ ). By Proposition 4.6 it follows that a symmetry $\varphi \in \operatorname{Sym} \Omega_{g, k}$ exists with $\varphi(x)=x^{\prime}$. Let $M(x)$ (resp. $M^{\prime}\left(x^{\prime}\right)$ ) be the hyperbolic structure defined by $x$ on $M$ (resp. by $x^{\prime}$ on $M^{\prime}$ ). Recall that $M(\mathscr{S})$ (resp. $M^{\prime}\left(\mathscr{S}^{\prime}\right)$ ) is isometric to the metric completion of $M(x)$ (resp. of $M^{\prime}\left(x^{\prime}\right)$ ), and that $M(\mathscr{S}) \backslash M(x)$ (resp. $\left.M^{\prime}\left(\mathscr{Y}^{\prime}\right) \backslash M^{\prime}\left(x^{\prime}\right)\right)$ is the union of $h$ disjoint geodesics in $M(\mathscr{S})$ (resp. in $M\left(\mathscr{S}^{\prime}\right)$ ). Notice that since $x$ is $\operatorname{Sym} \Omega_{g, k}$-equivalent to $x^{\prime}$, the geometric partially truncated tetrahedra in the decomposition of $M(x)$ are isometric to the geometric tetrahedra in the decomposition of $M^{\prime}\left(x^{\prime}\right)$. Together with Lemma 4.7, this readily implies that the shortest return paths of $M(\mathscr{Y})$ and $M\left(\mathscr{Y}^{\prime}\right)$ have the same length. Moreover, $M(x)$ and $M^{\prime}\left(x^{\prime}\right)$ have the same volume, whence $\operatorname{volume}(M(\mathscr{S}))=\operatorname{volume}\left(M^{\prime}\left(\mathscr{\varphi}^{\prime}\right)\right)$.

By Theorem 1.3 the bases of the cusps of $M(\mathscr{S})$ and $M^{\prime}\left(\mathscr{Y}^{\prime}\right)$ are all isometric to regular hexagonal tori, so $M(\mathscr{S})$ and $M^{\prime}\left(\mathscr{S}^{\prime}\right)$ share the same cusp shape.

Now consider the shape of the geometric tetrahedra in the triangulations

$$
\begin{aligned}
\mathscr{T} & =\left\{\Delta_{1}, \ldots, \Delta_{g+k}\right\}, \\
\mathscr{T}^{\prime} & =\left\{\Delta_{1}^{\prime}, \ldots, \Delta_{g+k}^{\prime}\right\}
\end{aligned}
$$

of $M, M^{\prime}$ respectively. Without loss of generality we can order the tetrahedra of these triangulations in such a way that $\Delta_{l}, \Delta_{l}^{\prime}$ are asymptotic to the cusps of $M(\mathscr{S}), M^{\prime}\left(\mathscr{S}^{\prime}\right)$ for $l=2 h+1, \ldots, 2 k$ (this is equivalent to requiring that the slopes in $\mathscr{S}$ and $\mathscr{S}^{\prime}$ lie on $T_{1}, \ldots, T_{h}$ and $T_{1}^{\prime}, \ldots, T_{h}^{\prime}$ ). Then by Proposition 3.5 a real number $\vartheta(x)=\vartheta\left(x^{\prime}\right) \in(0, \pi / 3)$ exists such that $\Delta_{l}$ and $\Delta_{l}^{\prime}$ are isometric to $\Delta^{\vartheta(x)}$ for $l=2 h+1, \ldots, 2 k$. For $l=2 h+1, \ldots, 2 k$ let now $v_{l}, v_{l}^{\prime}$ be the ideal vertices of $\Delta_{l}, \Delta_{l}^{\prime}$ respectively. The same argument as in Theorem 4.3 (1) shows that, up to increasing $C$, we can suppose that a unique horocusp neighbourhood $H_{l}$ of $v_{l}$ in $\Delta_{l}$ exists which is tangent to the truncation triangles of $\Delta_{l}$ and is entirely contained in $\Delta_{l}$. Moreover $H_{2 i-1}$ and $H_{2 i}$ glue up in $M(\mathscr{S})$ giving a horocusp neighbourhood $O_{i}$ of the $i$-th cusp for $i=h+1, \ldots, k$. Also notice that the total horocusp neighbourhood $O_{h+1} \sqcup \cdots \sqcup O_{k}$ is regular (since the $H_{l}$ 's are isometric to each other) and maximal (since each $O_{i}$ is tangent to the boundary of $M(\mathscr{Y})$ ). The 
very same construction also leads to a horocusp neighbourhood $O_{h+1}^{\prime} \sqcup \cdots \sqcup O_{k}^{\prime}$ for $M^{\prime}\left(\mathscr{S}^{\prime}\right)$. Since $\Delta_{l}$ is isometric to $\Delta_{l}^{\prime}$ for $l=2 h+1, \ldots, 2 k$, we have

$$
\operatorname{vol}\left(O_{h+1} \sqcup \cdots \sqcup O_{k}\right)=\operatorname{vol}\left(O_{h+1}^{\prime} \sqcup \cdots \sqcup O_{k}^{\prime}\right),
$$

so $M(\mathscr{S})$ and $M^{\prime}\left(\mathscr{Y}^{\prime}\right)$ share the same cusp volume.

Recall now that Thurston's hyperbolic Dehn filling Theorem ensures that if for all $l=1, \ldots, h$ we have $L\left(s_{l}\right)>C^{\prime}>0$ for some sufficiently large $C^{\prime}$, then the shortest geodesics of $M(\mathscr{S})$ and $M^{\prime}\left(\mathscr{S}^{\prime}\right)$ are exactly the geodesics added to $M(x)$ and $M^{\prime}\left(x^{\prime}\right)$. Thus under the hypothesis that $L\left(s_{l}\right)>C^{\prime}$ for all $l=1, \ldots, h$, in order to prove that the shortest geodesics of $M(\mathscr{S})$ and $M^{\prime}\left(\mathscr{Y}^{\prime}\right)$ have the same complex length we only have to compute the complex length of these added geodesics. The desired result is then easily obtained from the analysis of the action of Sym $\Omega_{g, k}$ on Dehn filling coefficients, together with equation (2-6).

The fact that $H_{1}(M(\mathscr{S}) ; \mathbb{Z})$ is isomorphic to $H_{1}\left(M^{\prime}\left(\mathscr{S}^{\prime}\right) ; \mathbb{Z}\right)$ is an immediate consequence of Proposition 4.4. By Theorem 4.5, if $\Sigma$ (resp. $\Sigma^{\prime}$ ) is the geodesic boundary of $M(\mathscr{Y})$ (resp. of $M^{\prime}\left(\mathscr{Y}^{\prime}\right)$ ), the Heegaard genus of both

$$
(M(\mathscr{S}), \Sigma, \partial M(\mathscr{S}) \backslash \Sigma) \quad \text { and } \quad\left(M^{\prime}\left(\mathscr{Y}^{\prime}\right), \Sigma^{\prime}, \partial M^{\prime}\left(\mathscr{Y}^{\prime}\right) \backslash \Sigma^{\prime}\right)
$$

is equal to $g+1$.

In order to prove our statement about Turaev-Viro invariants we need to construct ideal triangulations $\mathscr{T}(\mathscr{S})$ for $M(\mathscr{Y})$ and $\mathscr{T}^{\prime}\left(\mathscr{Y}^{\prime}\right)$ for $M^{\prime}\left(\mathscr{S}^{\prime}\right)$. Since $\mathscr{Y}$ is equivalent to $\mathscr{S}^{\prime}$, such triangulations can be chosen in such a way that the incidence numbers between edges and tetrahedra are the same for $\mathscr{T}(\mathscr{S})$ and for $\mathscr{T}^{\prime}\left(\mathscr{T}^{\prime}\right)$ (see [Frigerio 2005] for a detailed proof). As pointed out in [Matveev and Nowik 1994], this implies that $M(\mathscr{S})$ and $M^{\prime}\left(\mathscr{Y}^{\prime}\right)$ share the same Turaev-Viro invariants.

4F. Nonhomeomorphic fillings. It is explained in [Benedetti and Petronio 1995] how ideal triangulations of compact orientable 3-manifolds can be encoded by $o$ graphs. Let $\mathscr{T}_{k}$ be the ideal triangulation encoded by the o-graph described in Figure 2, and let $X_{k}$ be the manifold obtained by gluing the tetrahedra of $\mathscr{T}_{k}$. Computing the boundary of $X_{k}$ as explained in that paper, one can easily prove that $X_{k} \in \mathcal{M}_{k+1, k}$ if $k$ is odd and $X_{k} \in \mathcal{M}_{k+2, k}$ if $k$ is even. Moreover, $\mathscr{T}_{k}$ is the Kojima decomposition of $X_{k}$.

Proposition 4.9. For all $k \geqslant 1$, the manifold $X_{k}$ admits no nontrivial isometries.

Proof. Since $\mathscr{T}_{k}$ is the Kojima decomposition of $X_{k}$, the group of isometries of $X_{k}$ is canonically isomorphic to the group Aut $\mathscr{T}_{k}$ of the combinatorial automorphisms of $\mathscr{T}_{k}$. Now a straightforward analysis of the combinatorics of $\mathscr{T}_{k}$ shows that Aut $\mathscr{T}_{k}$ is trivial, whence the conclusion.

Together with Proposition 4.9, the following result implies Theorem 1.6. 
$C_{1}$

$C_{2}$

$C_{2 j+1}$

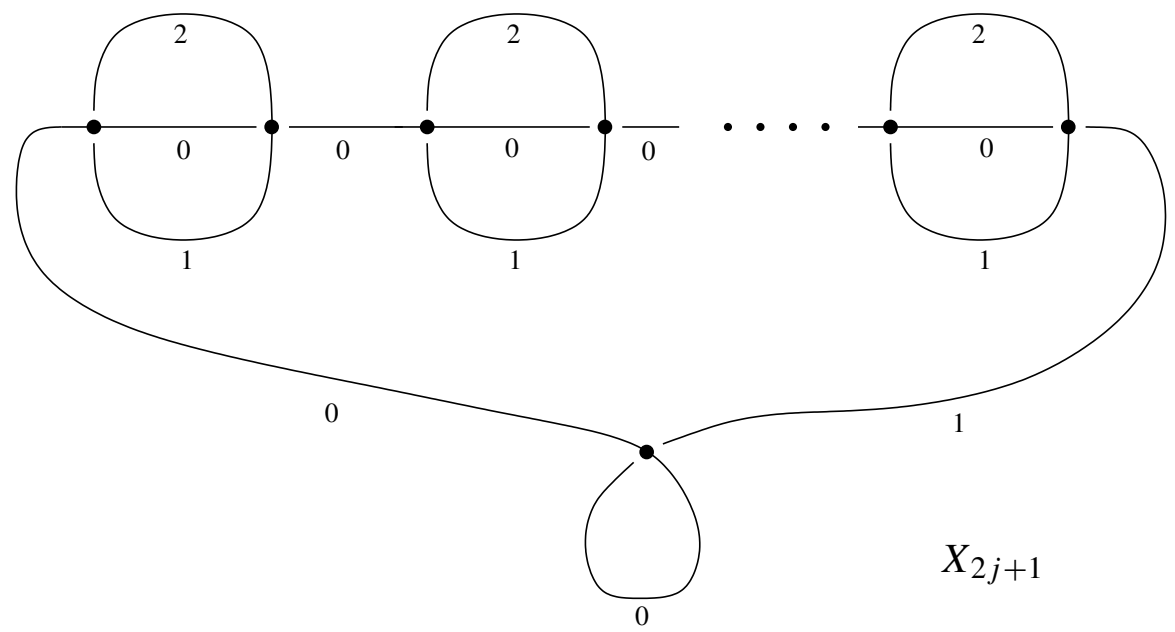

$C_{1}$

$C_{2}$

$C_{2 j+1}$

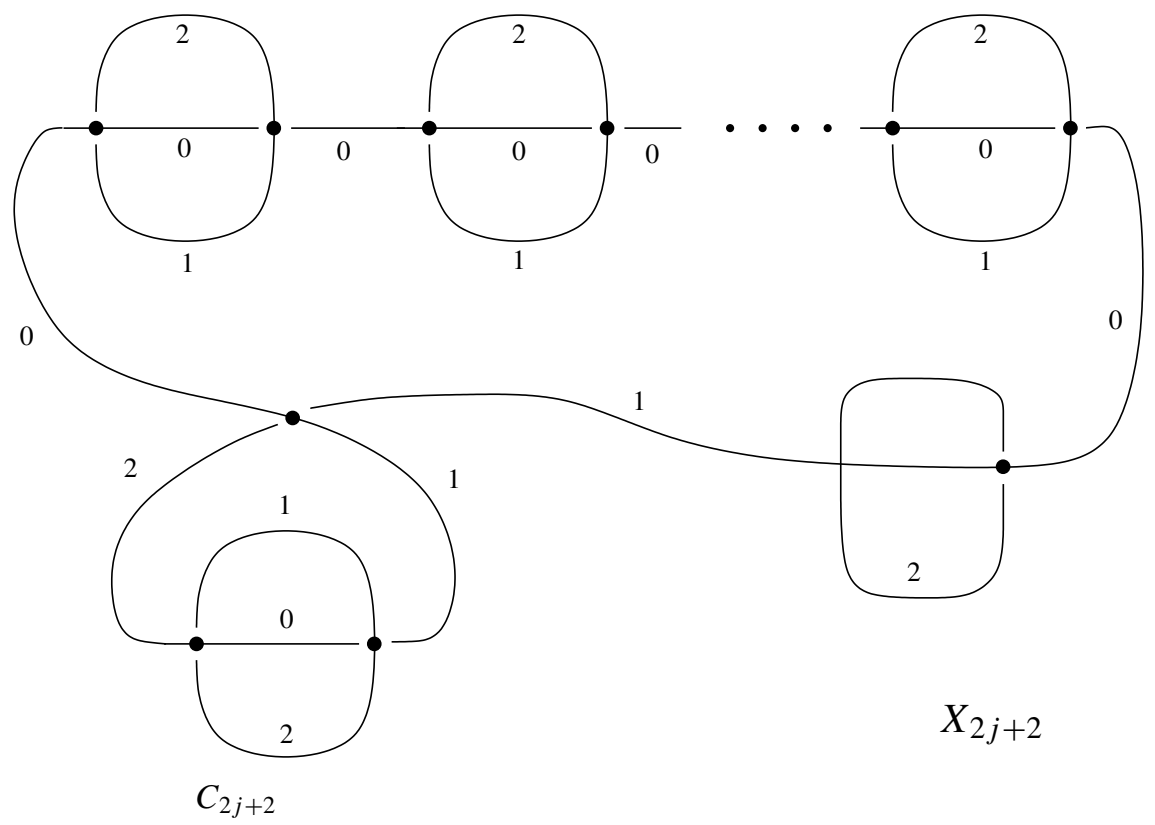

Figure 2. The o-graphs encoding the Kojima decomposition of $X_{k}$ (their combinatorics are different according to the fact that $k$ is either even or odd). Each pair of vertices joined by three edges gives rise to a toric cusp in $X_{k}$. 
Proposition 4.10. Let $X \in \mathcal{M}_{g, k}$ with boundary tori $T_{1}, \ldots, T_{k}$ and suppose that $X$ admits no nontrivial isometry. For each $i=1, \ldots, k$ we can choose a finite set $\mathscr{Y}_{i}$ of slopes on $T_{i}$ with the following property. Let $\mathscr{Y}$ be a set of slopes for $X$ whose elements do not belong to $\mathscr{Y}_{i}, i=1, \ldots, k$ and let $h=\# \mathscr{S} \leqslant k$. Then the number of sets of slopes equivalent to $\mathscr{Y}$ is greater than or equal to $\left(k ! \cdot 3^{h}\right) /(h ! \cdot(k-h) !)$. Moreover, if $\mathscr{S}^{\prime}$ is a set of slopes equivalent to $\mathscr{S}$ and $X(\mathscr{S})$ is homeomorphic to $X\left(\mathscr{Y}^{\prime}\right)$, then $\mathscr{Y}=\mathscr{Y}^{\prime}$.

Proof. Thurston's hyperbolic Dehn filling Theorem and Theorem 4.8 imply that we can choose the finite set $\mathscr{Y}_{i}$ in such a way that if $\mathscr{Y}$ is as in the statement and $\mathscr{S}^{\prime}$ is a set of slopes equivalent to $\mathscr{Y}$, then the following conditions hold: no slope in $\mathscr{S}^{\prime}$ is contained in some $\mathscr{Y}_{i} ; X(\mathscr{Y}), X\left(\mathscr{S}^{\prime}\right)$ are geometrically similar hyperbolic 3-manifolds; the cores of the added solid tori give the $h$ shortest geodesics both of $X(\mathscr{S})$ and of $X\left(\mathscr{S}^{\prime}\right)$.

An elementary combinatorial argument shows that the number of sets of slopes equivalent to $\mathscr{S}$ is at least $\left(k ! \cdot 3^{h}\right) /(h ! \cdot(k-h) !)$.

Now suppose that $\mathscr{S}^{\prime}$ is equivalent to $\mathscr{Y}$ and let $\psi: X(\mathscr{S}) \rightarrow X\left(\mathscr{S}^{\prime}\right)$ be a homeomorphism. By the Mostow-Prasad rigidity Theorem, $\psi$ is homotopic to an isometry $\psi^{\prime}$, which must take the added geodesics of $X(\mathscr{S})$ to the added geodesics of $X\left(\mathscr{S}^{\prime}\right)$. This gives in turn a homeomorphism of $X$ onto itself taking $\mathscr{Y}$ onto $\mathscr{Y}^{\prime}$. By rigidity again, up to homotopy such a homeomorphism restricts to an isometry of $X$, whence $\mathscr{Y}=\mathscr{Y}^{\prime}$ since $X$ admits no nontrivial isometry.

\section{Noncommensurable similar Dehn fillings}

Two complete hyperbolic $n$-manifolds with geodesic boundary $M_{1}, M_{2}$ are commensurable if a hyperbolic manifold with geodesic boundary $M_{3}$ exists which is the total space of a finite Riemannian covering both of $M_{1}$ and of $M_{2}$.

A commensurability criterion. Let $M \in \mathcal{M}_{g, k}$ with canonical decomposition $\mathscr{T}$. Let $N$ be a hyperbolic manifold obtained by Dehn filling $M$ along a sufficiently complicated slope, let $\widetilde{N}$ be the universal covering of $N$, and let $x \in I \Omega_{g, k}$ be such that $N \cong \widehat{M}(x)$. As in Section $4 \mathrm{D}$, we denote by $l_{x}$ the unique shortest return path in $N$, and by $L^{x}\left(l_{x}\right)$ the length of $l_{x}$. Building on Proposition 4.1 and on Lemma 4.7, one can show that there exists a neighbourhood $V$ of $x_{0}$ in $\Omega_{g, k}$ with the following property: let $x \in I \Omega_{g, k} \cap V$ and $S_{1}, \ldots, S_{4}$ be pairwise distinct connected components of $\tilde{N}$; then $d\left(S_{i}, S_{j}\right)=L^{x}\left(l_{x}\right)$ for all $i \neq j$ if and only if there exists a lift of a compact tetrahedron in the geometric triangulation parameterized by $x$ whose truncation triangles lie on $S_{1}, \ldots, S_{4}$.

Let now $p$ be a point on $l_{x}$, take a small geodesic surface $H$ passing through $p$ and orthogonal to $l_{x}$, and let $B_{\varepsilon} \subset H$ be the disc with center $p$ and radius $\varepsilon$. Let also $Z \subset M(x)$ be the union of all the compact tetrahedra of $\mathscr{T}$. The commensurability 
class of $N$ determines the isometry class of the universal covering $\widetilde{N}$, whence, by the discussion above, the isometry class of the pair $\left(B_{\varepsilon}, B_{\varepsilon} \cap Z\right)$. Of course, such isometry class only depends on the way in which the dihedral angles of the tetrahedra in $M(x)$ are arranged around $l_{x}$, and therefore can be explicitly described in terms of the combinatorics of $\mathcal{T}$ and of $x$.

Noncommensurable Dehn fillings. From now on, let $k$ be a fixed odd natural number, let $X_{k}$ be the manifold defined in Section 4F and $l \subset X_{k}$ be the compact edge of $\mathscr{T}_{k}$. If $x \in \Omega_{k+1, k}$, we denote by $X_{k}(x)$ the hyperbolic structure induced by $x$ on $X_{k}$, and by $\widehat{X}_{k}(x)$ the metric completion of $X_{k}(x)$. A straight-forward analysis of the combinatorics of $\mathscr{T}_{k}$ shows that the dihedral angles of the geometric tetrahedra of $\mathscr{T}_{k}$ parameterized by $x$ are arranged along $l$ according to the cyclic ordering

$$
\begin{array}{ll}
x_{12 k+1}, & x_{1}, x_{7}, x_{13}, \ldots, x_{6 l+1}, \ldots, x_{12 k-5}, \\
x_{12 k+1}, x_{12 k+1}, & x_{2}, x_{8}, x_{14}, \ldots, x_{6 l+2}, \ldots, x_{12 k-4}, \\
x_{12 k+1}, x_{12 k+1}, x_{12 k+1}, & x_{3}, x_{9}, x_{15}, \ldots, x_{6 l+3}, \ldots, x_{12 k-3} .
\end{array}
$$

Let $a, b, c: \Omega_{k+1, k} \rightarrow \mathbb{R}$ be the functions defined by

$$
a(x)=\sum_{i=0}^{2 k-1} x_{6 i+1}, \quad b(x)=\sum_{i=0}^{2 k-1} x_{6 i+2}, \quad c(x)=\sum_{i=0}^{2 k-1} x_{6 i+3} .
$$

The discussion carried out in Section 5 readily implies the following:

Proposition 5.1. Let $V$ be a sufficiently small neighbourhood of $x_{0}$ in $\Omega_{k+1, k}$, let $x, x^{\prime}$ be points in $I \Omega_{k+1, k} \cap V$, and suppose that $\widehat{X}_{k}(x)$ is commensurable with $\widehat{X}_{k}\left(x^{\prime}\right)$. Then $a(x)=a\left(x^{\prime}\right), b(x)=b\left(x^{\prime}\right), c(x)=c\left(x^{\prime}\right)$.

To determine if geometrically similar manifolds obtained by Dehn filling $X_{k}$ are commensurable with each other, we're left with the task of determining when the functions $a, b$ and $c$ take different values on Sym $\Omega_{k+1, k}$-equivalent points in $\Omega_{k+1, k}$. To this end, set

$$
H=\left\{x \in \mathbb{R}^{12 k+1}: x_{12 i+1}=x_{12 i+2}=x_{12 i+3} \text { for all } i=1, \ldots, k-1\right\} .
$$

We recall that in a neighbourhood of $x_{0}$ in $\Omega_{k+1, k}$ the set $\Omega_{k+1, k}^{*}:=H \cap \Omega_{k+1, k}$ is a smooth 2-dimensional manifold whose points correspond to those structures which induce a complete metric on all but the first cusp of $X_{k}$ (see Lemma 3.4 and Proposition 3.7).

In what follows, we denote simply by $\bar{\alpha}, \bar{\beta}$ the angles $\bar{\alpha}_{k+1, k}, \bar{\beta}_{k+1, k}$. By Proposition 3.3 the subspace of $\mathbb{R}^{12 k+1}$ having equations $\left\{x \in \mathbb{R}^{12 k+1}: x_{2}=x_{3}, x_{12 i+1}=\right.$ $\left.x_{12 i+2}=x_{12 i+3}, i=1, \ldots, k-1\right\}$ intersects $\Omega_{k+1, k}^{*}$ transversely near $x_{0}$ in the 
support of a smooth curve $\bar{\rho}:(-\varepsilon, \varepsilon) \rightarrow \Omega_{k+1, k}$ with $\bar{\rho}(0)=x_{0}$ and

$$
\begin{aligned}
\dot{\bar{\rho}}(0)= & (2 \sin \bar{\alpha},-\sin \bar{\alpha},-\sin \bar{\alpha}, 2 \sqrt{3} \cos \bar{\alpha},-\sqrt{3} \cos \bar{\alpha},-\sqrt{3} \cos \bar{\alpha}, \\
& -2 \sin \bar{\alpha}, \sin \bar{\alpha}, \sin \bar{\alpha},-2 \sqrt{3} \cos \bar{\alpha}, \sqrt{3} \cos \bar{\alpha}, \sqrt{3} \cos \bar{\alpha}, 0, \ldots, 0) .
\end{aligned}
$$

A long computation [Frigerio 2005] shows that we have

$$
\ddot{\bar{\rho}}(0)=(8 \cos \bar{\alpha} \sin \bar{\alpha},-4 \cos \bar{\alpha} \sin \bar{\alpha},-4 \cos \bar{\alpha} \sin \bar{\alpha}, 2 \sqrt{3},-\sqrt{3},-\sqrt{3},
$$

$8 \cos \bar{\alpha} \sin \bar{\alpha},-4 \cos \bar{\alpha} \sin \bar{\alpha},-4 \cos \bar{\alpha} \sin \bar{\alpha}, 2 \sqrt{3},-\sqrt{3},-\sqrt{3}, 0, \ldots, 0)$.

For a smooth $f: \Omega_{k+1, k} \rightarrow \mathbb{R}$, denote by $\dot{f}$ and $\ddot{f}$ the first and second derivatives of $f \circ \bar{\rho}$ at 0 . From equations (5-1) and (5-2) we deduce that $\dot{a}=\dot{b}=\dot{c}=0$, while $\ddot{a}>\ddot{b}>\ddot{c}$.

Theorem 5.2. Let $k$ be odd. There exists a sequence $\left\{W_{n}\right\}_{n \in \mathbb{N}}$ of pairwise nonhomeomorphic complete finite-volume hyperbolic manifolds with geodesic boundary with the following properties:

- Each $W_{n}$ is obtained by Dehn filling the first cusp of $X_{k}$;

- For any natural number $n$ there exist at least three (including $W_{n}$ itself) pairwise noncommensurable hyperbolic Dehn fillings of $X_{k}$ that are geometrically similar to $W_{n}$.

Proof. We choose an infinite sequence $\left\{y_{n}\right\}_{n \in \mathbb{N}} \subset I \Omega_{k+1, k}^{*} \backslash\left\{x_{0}\right\}$ converging to $x_{0}$ along $\dot{\bar{\rho}}(0)$ (see Definition 3.9), and we set $W_{n}=\widehat{X}_{k}\left(y_{n}\right)$.

First note that, since $y_{n}$ belongs to $\Omega_{k+1, k}^{*}$, the last $k-1$ cusps of $X_{k}\left(y_{n}\right)$ have to be complete, so $W_{n}$ is obtained from $X_{k}$ by Dehn filling the first cusp of $X_{k}$.

Let $r \in \mathcal{M}\left(T_{1} \sqcup \cdots \sqcup T_{k}\right)$ be the element acting as a positive rotation by angle of $\pi / 3$ on $T_{1}$, and as the identity on $T_{i}$ for $i \in\{2, \ldots, k\}$. If

$$
\Theta: M\left(T_{1} \sqcup \cdots \sqcup T_{k}\right) \rightarrow \operatorname{Sym} \Omega_{k+1, k}
$$

is the isomorphism described in Proposition 4.6, we set $y_{n}^{\prime}=\Theta(r)\left(y_{n}\right)$ and $y_{n}^{\prime \prime}=$ $\Theta\left(r^{2}\right)\left(y_{n}\right)$. By construction, $W_{n}=\widehat{X}_{k}\left(y_{n}\right), \widehat{X}_{k}\left(y_{n}^{\prime}\right)$ and $\widehat{X}_{k}\left(y_{n}^{\prime \prime}\right)$ are pairwise geometrically similar. An easy computation shows that for $x \in \Omega_{k+1, k}$ we have

$$
\begin{aligned}
& a\left(\Theta\left(r^{2}\right)(x)\right)=c(\Theta(r)(x))=b(x), \\
& b\left(\Theta\left(r^{2}\right)(x)\right)=a(\Theta(r)(x))=c(x), \\
& c\left(\Theta\left(r^{2}\right)(x)\right)=b(\Theta(r)(x))=a(x),
\end{aligned}
$$

whence $a\left(y_{n}\right)>a\left(y_{n}^{\prime \prime}\right)>a\left(y_{n}^{\prime}\right)$, and $W_{n}=\widehat{X}_{k}\left(y_{n}\right), \widehat{X}_{k}\left(y_{n}^{\prime}\right), \widehat{X}_{k}\left(y_{n}^{\prime \prime}\right)$ are pairwise noncommensurable by Proposition 5.1.

Remark 5.3. Let $M$ be an element of $\mu_{g, k}$ with canonical decomposition $\mathscr{T}$. Suppose that the arrangement of compact and noncompact tetrahedra around the 
compact edge of $\mathscr{T}$ is sufficiently irregular and let $\mathscr{Y}=\left\{s_{i_{1}}, \ldots, s_{i_{h}}\right\}$ be a set of slopes for $M$ such that $s_{i_{l}}$ is not equivalent to $s_{i_{m}}$ for $l \neq m$. The same argument used to prove Theorem 5.2 shows that the Dehn fillings of $M$ which are geometrically similar to $M(\mathscr{Y})$ are expected to be noncommensurable with each other.

\section{Acknowledgements}

The results described in this paper are contained in my doctoral thesis. I would like to thank Prof. Carlo Petronio, my thesis advisor, for his support and encouragement.

\section{References}

[Benedetti and Petronio 1992] R. Benedetti and C. Petronio, Lectures on hyperbolic geometry, Universitext, Springer, Berlin, 1992. MR 94e:57015 Zbl 0768.51018

[Benedetti and Petronio 1995] R. Benedetti and C. Petronio, "A finite graphic calculus for 3-manifolds”, Manuscripta Math. 88:3 (1995), 291-310. MR 96m:57008 Zbl 0856.57009

[Calegari 2001] D. Calegari, "Napoleon in isolation", Proc. Amer. Math. Soc. 129:10 (2001), 31093119. MR 2002e:57023 Zbl 0971.57022

[Frigerio 2005] R. Frigerio, Deforming triangulations of hyperbolic 3-manifolds with geodesic boundary, Ph.D. thesis, Scuola Normale Superiore, 2005, Available at http://www.dm.unipi.it/ frigerio/ articoli/main.ps.

[Frigerio 2006] R. Frigerio, "On deformations of hyperbolic 3-manifolds with geodesic boundary", Algebr. Geom. Topol. 6 (2006), 435-457. MR MR2220684 Zbl 05029608

[Frigerio and Petronio 2004] R. Frigerio and C. Petronio, "Construction and recognition of hyperbolic 3-manifolds with geodesic boundary”, Trans. Amer. Math. Soc. 356:8 (2004), 3243-3282. MR 2005f:57025 Zbl 1052.57018

[Frigerio et al. 2003] R. Frigerio, B. Martelli, and C. Petronio, "Dehn filling of cusped hyperbolic 3manifolds with geodesic boundary", J. Differential Geom. 64:3 (2003), 425-455. MR 2005d:57024 Zbl 1073.57010

[Fujii 1990] M. Fujii, "Hyperbolic 3-manifolds with totally geodesic boundary which are decomposed into hyperbolic truncated tetrahedra", Tokyo J. Math. 13:2 (1990), 353-373. MR 92a:57043 Zbl 0729.57005

[Fujii 1992] M. Fujii, "On totally geodesic boundaries of hyperbolic 3-manifolds", Kodai Math. J. 15:2 (1992), 244-257. MR 93i:57018 Zbl 0777.53037

[Fujii 1993] M. Fujii, "Deformations of a hyperbolic 3-manifold not affecting its totally geodesic boundary”, Kodai Math. J. 16:3 (1993), 441-454. MR 94i:57023 Zbl 0809.52023

[Fujii and Kojima 1997] M. Fujii and S. Kojima, "Flexible boundaries in deformations of hyperbolic 3-manifolds”, Osaka J. Math. 34:3 (1997), 541-551. MR 99a:57013 Zbl 0887.57016

[Hodgson et al. 1992] C. D. Hodgson, G. R. Meyerhoff, and J. R. Weeks, "Surgeries on the Whitehead link yield geometrically similar manifolds", pp. 195-206 in Topology '90 (Columbus, OH, 1990), edited by B. N. Apanasov, Ohio State Univ. Math. Res. Inst. Publ. 1, de Gruyter, Berlin, 1992. MR 93i:57019 Zbl 0767.57007

[Kent 2005] R. P. Kent, "Totally geodesic boundaries of knot complements", Proc. Amer. Math. Soc. 133:12 (2005), 3735-3744. MR 2006e:57025 Zbl 1087.57012 
[Kojima 1990] S. Kojima, "Polyhedral decomposition of hyperbolic manifolds with boundary", pp. 37-57 in On the geometric structure of manifolds, edited by D. P. Chi, Proceedings of Workshops in Pure Math. 10:III, Seoul National University, 1990.

[Matveev and Nowik 1994] S. V. Matveev and T. Nowik, "On 3-manifolds having the same TuraevViro invariants", Russian J. Math. Phys. 2:3 (1994), 317-324. MR 96a:57043 Zbl 0911.57014

[Neumann and Reid 1993] W. D. Neumann and A. W. Reid, "Rigidity of cusps in deformations of hyperbolic 3-orbifolds", Math. Ann. 295:2 (1993), 223-237. MR 94c:57025 Zbl 0813.57013

[Neumann and Zagier 1985] W. D. Neumann and D. Zagier, "Volumes of hyperbolic three-manifolds", Topology 24:3 (1985), 307-332. MR 87j:57008 Zbl 0589.57015

[Thurston 1979] W. P. Thurston, “The geometry and topology of three-manifolds", lecture notes, Princeton University, 1979, Available at http://msri.org/publications/books/gt3m.

[Turaev and Viro 1992] V. G. Turaev and O. Y. Viro, "State sum invariants of 3-manifolds and quantum 6j-symbols”, Topology 31:4 (1992), 865-902. MR 94d:57044 Zbl 0779.57009

Received July 15, 2005.

\author{
ROBERTO FRIGERIO \\ DiPARTIMENTO Di MATEMATICA \\ UNIVERSITÀ DI PISA \\ LARGO B. PONTECORVO 5 \\ 56127 PISA \\ ITALY \\ frigerio@dm.unipi.it \\ http://www.dm.unipi.it/ frigerio/
}

